\title{
Robust Tests for a Linear Trend with an Application to Equity Indices*
}

\author{
Sam Astill ${ }^{a}$, David I. Harvey ${ }^{b}$, Stephen J. Leybourne ${ }^{b}$ and A.M. Robert Taylor ${ }^{c}$ \\ a. Department of Economics, University of Warwick. \\ b. Granger Centre for Time Series Econometrics and School of Economics, University of Nottingham. \\ c. Essex Business School, University of Essex.
}

February 2014

\begin{abstract}
In this paper we develop a testing procedure for the presence of a deterministic linear trend in a univariate time series which is robust to whether the series is $\mathrm{I}(0)$ or $\mathrm{I}(1)$ and requires no knowledge of the form of weak dependence present in the data. Our approach is motivated by the testing procedures of Vogelsang [1998, Econometrica, vol 66, p123-148] and Bunzel and Vogelsang [2005, Journal of Business and Economic Statistics, vol 23, p381-394], but utilises an auxiliary unit root test to switch between critical values in the exact $\mathrm{I}(1)$ and $\mathrm{I}(0)$ environments, rather than using this unit root test to scale the test statistic as is done in the aforementioned procedures. We show that our proposed tests have uniformly greater local asymptotic power than the tests of Vogelsang (1998) and Bunzel and Vogelsang (2005) when the error process is exact I(1), identical local asymptotic when the error process is $\mathrm{I}(0)$, and have better overall local asymptotic power when the error process is near $\mathrm{I}(1)$. Our proposed tests also display superior finite sample power to the tests of Vogelsang (1998) and Bunzel and Vogelsang (2005) and are competitive in finite samples with tests designed to be optimal in both the exact $\mathrm{I}(1)$ and $\mathrm{I}(0)$ environments. We apply our test procedures to a number of equity indices and find that these series appear to have a significant upward deterministic trend, yet are also highly persistent about this long run growth path.
\end{abstract}

Keywords: Linear trend, unit root tests, strong serial correlation.

JEL Classification: C22.

\footnotetext{
${ }^{*}$ We thank the Guest Editors, Richard Baillie and Menelaos Karanasos, and two anonymous referees for helpful comments on earlier versions of the paper. Address correspondence to: Robert Taylor, Essex Business School, University of Essex, Colchester, CO4 3SQ, UK. E-mail: rtaylor@essex.ac.uk
} 


\section{Introduction}

The ability to detect the presence and magnitude of a deterministic trend in an economic time series is of key importance when conducting empirical analysis, the presence of a linear trend being of particular relevance for the purpose of forecasting or testing for the presence of a unit root. In the latter case, failure to correctly specify a trend when it is indeed present is known to have an adverse effect, resulting in non-similar and inconsistent tests, as demonstrated by Perron (1998). Similarly, the power of unit root tests to reject the null under the $\mathrm{I}(0)$ alternative when a trend is unnecessarily included in a model specification is reduced, see inter alia Marsh (2009) and Ellliot et al. (1996). The presence of a deterministic trend in an economic or financial time series can also be of interest in its own right, since a linear trend is compatible with a degree of underlying long run growth in the series. For example, this is of particular interest when considering the long run behaviour of stock prices and indices, where an underlying upward trend implies a long run average return. Moreover, the outcome of statistical tests of the efficient market hypothesis (EMH) are necessarily contingent on correct specification of the trend component of prices (or, equivalently, the mean component of returns).

Testing for the presence of a linear trend is complicated by the fact that in practice it is typically not known whether the underlying process is $\mathrm{I}(0)$ or $\mathrm{I}(1)$. For example, uncertainty as to the degree to which financial markets are efficient suggests that one would not want to make an a priori assumption regarding the presence or absence of a unit root in the series from the outset. We therefore require tests for a linear trend that are robust to whether a series is $\mathrm{I}(0)$ or $\mathrm{I}(1)$ when determining whether a trend is present. There have been a number of papers suggesting testing procedures for detecting a deterministic trend function which are robust to the order of integration of the data including, inter alia, Vogelsang (1998), Bunzel and Vogelsang (2005), Harvey et al. (2007) and Perron and Yabu (2009). Vogelsang (1998) and Bunzel and Vogelsang (2005) employ an auxiliary unit root test statistic to line up the critical values of a $t$-statistic based on the levels of the data in the exact I(1) and I(0) environments. The approach taken in Harvey et al. (2007) utilises an auxiliary unit root or stationary test statistic to switch between the optimal trend function test in the exact $\mathrm{I}(1)$ and $\mathrm{I}(0)$ environments, and, as such, the test achieves the Gaussian asymptotic local power envelope in both cases. Perron and Yabu (2009) use a "super-efficient" estimate of the autoregressive parameter to construct a GLS based test statistic that also achieves the Gaussian asymptotic local power envelope in both the exact $\mathrm{I}(1)$ and $\mathrm{I}(0)$ cases.

Compared to the tests of Harvey et al. (2007) and Perron and Yabu (2009), the tests of Vogelsang (1998) and Bunzel and Vogelsang (2005) have the advantage of better size control in finite samples in the exact I(1) environment when the errors are i.i.d., albeit at the expense of relatively poor power properties, with the tests of Harvey et al. (2007) and Perron and Yabu (2009) fairly similar in terms of their overall performance. In the local to I(1) environment the results are less clear, with all tests displaying significant undersize, and no one test dominating in terms of overall power.

In this paper we propose a modification to the testing procedures of Vogelsang (1998) and Bunzel 
and Vogelsang (2005) in which an auxiliary unit root test statistic is used to scale the critical value of the test rather than the test statistic itself. We find the proposed modification yields a test that has uniformly greater local asymptotic power than the tests of Vogelsang (1998) and Bunzel and Vogelsang (2005) when the error process is exact I(1), has identical local asymptotic power when the error process is $\mathrm{I}(0)$ and has better overall finite sample properties. We also find that the proposed tests are competitive in finite samples when compared to the optimal tests of Harvey et al. (2007) and Perron and Yabu (2009).

The paper is organised as follows. Section 2 outlines the model. Extant tests for a deterministic linear trend are outlined in Section 3. In section 4 we outline our proposed tests. In section 5 the limiting distribution and local asymptotic power of the tests are detailed. Section 6 reports results of Monte Carlo simulations performed in order to assess the finite sample size and power properties of the proposed tests relative to existing tests. Section 7 reports results of an empirical exercise in which we apply the test statistics outlined in this paper to a number of US and UK equity indices. Concluding remarks are made in section 8 .

\section{The Linear Trend Model}

Consider a sample of $T$ observations generated according to the following data generating process (DGP)

$$
\begin{aligned}
& y_{t}=\mu+\beta t+u_{t}, \quad t=1, \ldots, T \\
& u_{t}=\alpha_{T} u_{t-1}+\varepsilon_{t}, \quad t=2, \ldots, T .
\end{aligned}
$$

Following Vogelsang (1998), Bunzel and Vogelsang (2005) and Harvey et al. (2007) we assume that the process $\left\{\varepsilon_{t}\right\}$ is such that

$$
\varepsilon_{t}=C(L) e_{t}, C(L):=\sum_{i=0}^{\infty} C_{i} L^{i}
$$

with $C(z) \neq 0$ for all $|z| \leq 1$ and $\sum_{i=0}^{\infty} i\left|C_{i}\right|<\infty$, and where $\left\{e_{t}, \mathcal{F}_{t}\right\}$ is a martingale difference sequence with $E\left(e_{t}^{2} \mid \mathcal{F}_{t-1}\right)=1$ and $\sup _{t} E\left(e_{t}^{4} \mid \mathcal{F}_{t-1}\right)<\infty$. We also define $\omega_{\varepsilon}^{2}:=\lim _{T \rightarrow \infty} T^{-1} E\left(\sum_{t=1}^{T} \varepsilon_{t}\right)^{2}=$ $C(1)^{2}$. The initial condition, $u_{1}$, is assumed to be $O_{p}(1)$. These assumptions ensure that we can apply a Functional Central Limit Theorem (FCLT) to the partial sums of $\left\{\varepsilon_{t}\right\}$, so that $T^{-1 / 2} \sum_{t=1}^{\lfloor r T\rfloor} \varepsilon_{t} \stackrel{d}{\rightarrow}$ $\omega_{\varepsilon} w(r)$ where $\lfloor$.$\rfloor denotes the integer part of its argument, \stackrel{d}{\rightarrow}$ denotes weak convergence and $w(r)$ is a standard Wiener process.

The autoregressive parameter in (2) determines the order of integration of the series. When $\alpha_{T}=\alpha$, and $|\alpha|<1$ the series is $\mathrm{I}(0)$, whereas if $\alpha_{T}=1-\bar{\alpha} / T$ the series is near I(1), with $\bar{\alpha}=0$ corresponding to an exact I(1) process. Under these assumptions a FCLT applies to the partial sum of $\left\{u_{t}\right\}$ defined as $S_{t}:=\sum_{j=1}^{t} u_{j}$. When $\left\{u_{t}\right\}$ is $\mathrm{I}(0), T^{-1 / 2} S_{\lfloor r T\rfloor} \stackrel{d}{\rightarrow} \sigma w(r)$, where $\sigma^{2}:=C(1)^{2} /(1-\alpha)^{2}$. When $\left\{u_{t}\right\}$ is near I(1), $T^{-1 / 2} u_{\lfloor r T\rfloor} \stackrel{d}{\rightarrow} \omega_{\varepsilon} w_{\bar{\alpha}}(r)$, where $w_{\bar{\alpha}}(r):=\int_{0}^{r} \exp (-\bar{\alpha}(r-s)) d w(s)$.

The null hypothesis of interest is $H_{0}: \beta=\beta_{0}$. This null hypothesis can be tested against either 
the two-sided alternative $H_{1}: \beta \neq \beta_{0}$, or against either the right-tailed alternative $H_{1}^{\prime}: \beta>\beta_{0}$ or the left tailed alternative $H_{1}^{\prime \prime}: \beta<\beta_{0}$. The leading case of interest is where $\beta_{0}=0$, so that the null and alternative hypotheses signify the absence or presence of a linear trend, respectively. When analysing the asymptotic performance of the tests outlined in this paper it will prove useful to consider the local alternative hypotheses of $H_{10}: \beta=\beta_{0}+\kappa T^{-3 / 2}$ and $H_{11}: \beta=\beta_{0}+\kappa T^{-1 / 2}$, where $\kappa$ is a finite constant, with the scalings $T^{-3 / 2}$ and $T^{-1 / 2}$ providing the appropriate Pitman drifts under $\mathrm{I}(0)$ and I(1) errors, respectively.

\section{Extant Tests}

In this section we outline the trend tests of Vogelsang (1998), Bunzel and Vogelsang (2005), Harvey et al. (2007) and Perron and Yabu (2009), all of which are designed to be robust to both the order of integration of the error process and weak dependence in the shocks.

\subsection{Scaled Test Statistics}

The $t P S W^{1}$ statistic of Vogelsang (1998) takes the form

$$
t P S W^{1}:=\frac{\hat{\beta}-\beta_{0}}{\sqrt{T^{-1} 100 s_{z}^{2}}} \exp \left(-c_{\xi} J\right)
$$

where $\hat{\beta}$ is the OLS estimator of $\beta$ from (1) and $J$ denotes the $J$ unit root test statistic of Park (1990) and Park and Choi (1988) given by the standard OLS Wald statistic normalised by $T^{-1}$ for testing the joint hypothesis $\gamma_{2}=\gamma_{3}=\ldots=\gamma_{9}$ in the following regression

$$
y_{t}=\alpha+\beta t+\sum_{i=2}^{9} \gamma_{i} t^{i}+u_{t} .
$$

The variance estimator is calculated as $s_{z}^{2}:=T^{-1} \sum_{t=1}^{T} \tilde{S}_{t}^{2}$ where $\tilde{S}_{t}$ denotes the residuals from the following regression

$$
z_{t}=\tilde{\mu} t+\tilde{\beta} \sum_{j=1}^{t} j+\tilde{S}_{t}, \quad t=1, \ldots, T
$$

where $z_{t}:=\sum_{s=1}^{t} y_{s}$.

The $t P S W^{1}$ statistic has a non-degenerate limiting distribution under both $\mathrm{I}(0)$ and $\mathrm{I}(1)$ errors. The motivation behind this approach is that if the errors are $\mathrm{I}(0), J \stackrel{p}{\rightarrow} 0$, leaving the asymptotic distribution of the statistic unaffected by the scaling factor $\exp \left(-c_{\xi} J\right)$, whereas if the errors are near $\mathrm{I}(1), J$ converges to a well defined limiting distribution, allowing the practitioner to choose a value of $c_{\xi}$ that lines up the asymptotic critical values of the test in the $\mathrm{I}(0)$ and exact $\mathrm{I}(1)$ environments for a given significance level, $\xi$.

The Dan-J statistic of Bunzel and Vogelsang (2005) is a modified version of the $t P S W^{1}$ test statistic of Vogelsang (1998) that employs a long run variance estimator based on the fixed- $b$ asymptotics of 
Kiefer and Vogelsang (2005). Specifically, the statistic is given by

$$
\text { Dan- } J:=\frac{\hat{\beta}-\beta_{0}}{\sqrt{\hat{\omega}_{u}^{2} / \sum_{t=1}^{T}(t-\bar{t})^{2}}} \exp \left(-c_{\xi}^{\prime} J\right)
$$

where the long run variance estimator, $\hat{\omega}_{u}^{2}$, is constructed using the Daniell kernel with a datadependent bandwidth. The bandwidth is given by $\max (b T, 2)$, where $b=b_{\text {opt }}(\hat{\bar{\alpha}})$, in which $\hat{\bar{\alpha}}:=$ $T(1-\hat{\alpha})$ with $\hat{\alpha}$ obtained by OLS estimation of (1) and (2), and $b_{\mathrm{opt}}($.$) is a step function given in$ Bunzel and Vogelsang (2005). The test statistic is, again, scaled by a function of the $J$ unit root test statistic of Park (1990) and Park and Choi (1988). The constant $c_{\xi}^{\prime}$ is, as in Vogelsang (1998), chosen such that for a given significance level $\xi$, Dan-J has the same asymptotic critical value under both $I(0)$ and exact $I(1)$ errors. The value of $c_{\xi}^{\prime}$ depends on $b$; Bunzel and Vogelsang (2005) provide a response surface for determining $c_{\xi}^{\prime}$ for a given significance level, and $b$. The critical values for the test also depend on $b$, and again a response surface is provided by the authors for a variety of significance levels. Because $\bar{\alpha}$ is not consistently estimated by $\hat{\bar{\alpha}}$, Bunzel and Vogelsang (2005) only provide a limiting distribution for Dan- $J$ when it is assumed that $\bar{\alpha}$ is known in the calculation of $b$; that is, when $b_{\text {opt }}(\hat{\bar{\alpha}})$ is replaced by $b_{\text {opt }}(\bar{\alpha})$. Although this strictly means that their asymptotic results are based on the limiting behaviour of an infeasible test, for the purposes of making comparisons tractable, in what follows the limit distribution for Dan-J is that using $b_{\text {opt }}(\bar{\alpha})$.

\subsection{Asymptotically Optimal Tests}

The $z_{\lambda}$ statistic of Harvey et al. (2007) employs a switching-based strategy that attains the local limiting Gaussian power envelope for this testing problem irrespective of whether $u_{t}$ is an exact $I(1)$ process or is $I(0)$. The test statistic is asymptotically distributed as a standard normal under the null in both cases. It is calculated as

$$
z_{\lambda}:=\left(1-\lambda^{*}\right) z_{0}+\lambda^{*} z_{1}
$$

where

$$
z_{0}:=\frac{\hat{\beta}-\beta_{0}}{\sqrt{\hat{\omega}_{u}^{2} / \sum_{t=1}^{T}(t-\bar{t})^{2}}} \quad \text { and } \quad z_{1}:=\frac{\check{\beta}-\beta_{0}}{\sqrt{\check{\omega}_{v}^{2} /(T-1)}}
$$

and where $\hat{\beta}$ denotes the OLS estimator of $\beta$ from (1) and $\hat{\omega}_{u}^{2}$ is a long run variance estimator formed from $\hat{u}_{t}:=y_{t}-\hat{\mu}-\hat{\beta}$, while $\check{\beta}$ is the OLS estimator of $\beta$ from (1) estimated in first differences i.e. from $\Delta y_{t}=\beta+v_{t}, t=2, \ldots, T$, and $\check{\omega}_{v}^{2}$ is a long run variance estimator based on $\check{v}_{t}:=\Delta y_{t}-\check{\beta}$. The weight function $\lambda^{*}$ is defined as

$$
\lambda^{*}:=\exp \left(-0.00025\left(\frac{\mathrm{DF}-\mathrm{GLS}^{\tau}}{K P S S}\right)^{2}\right)
$$

where DF-GLS ${ }^{\tau}$ is the with-trend local GLS unit root test statistic of Elliott et al. (1996) and KPSS is the with-trend stationarity test statistic of Kwiatkowski et al. (1992). 
Whilst the test based on $z_{\lambda}$ attains the local limiting Gaussian power envelope for the case of either $\mathrm{I}(0)$ or exact I(1) errors, Harvey et al. (2007) show that a modified variant of $z_{\lambda}$, denoted $z_{\lambda}^{m 2}$, can provide a more powerful test than $z_{\lambda}$ when $u_{t}$ is near-integrated. This replaces $z_{1}$ with $z_{1}^{m 2}:=\delta_{\xi} R_{2} z_{1}$ where

$$
R_{2}:=\left(\frac{\check{\omega}_{v}^{2}}{T^{-1} \hat{\sigma}_{u}^{2}}\right)^{2}
$$

and $\hat{\sigma}_{u}^{2}:=(T-2)^{-1} \sum_{t=1}^{T} \hat{u}_{t}^{2}$. Here $\delta_{\xi}$ is a constant chosen such that, at a given significance level $\xi$, $z_{\lambda}^{m 2}$ has a standard normal critical value under both $\mathrm{I}(0)$ and exact $\mathrm{I}(1)$ errors.

The $t_{\beta}^{R Q F}$ test statistic of Perron and Yabu (2009) takes the form of an autocorrelation-corrected $t$-ratio on the OLS estimate of $\beta$ obtained from the quasi GLS regression

$$
\begin{aligned}
y_{t}-\tilde{\alpha}_{M S} y_{t-1} & =\left(1-\tilde{\alpha}_{M S}\right) \mu+\beta\left[t-\tilde{\alpha}_{M S}(t-1)\right]+\left(u_{t}-\tilde{\alpha}_{M S} u_{t-1}\right), \quad t=2, \ldots, T \\
y_{1} & =\beta+u_{1} .
\end{aligned}
$$

Here, $\tilde{\alpha}_{M S}$ is defined according to the following truncation rule

$$
\tilde{\alpha}_{M S}:= \begin{cases}1 & \text { if }\left|\tilde{\alpha}_{T W S}-1\right|<T^{-1 / 2} \\ \tilde{\alpha}_{T W S} & \text { otherwise }\end{cases}
$$

where $\tilde{\alpha}_{T W S}$ is an autocorrelation-robust weighted symmetric least squares estimate of $\alpha$ (based on the OLS residuals $\hat{u}_{t}$ ) with one of two truncations applied as described by Roy and Fuller (2001) and Roy et al. (2004). We will concentrate only on the truncation rule $\tilde{\alpha}_{T W S}=\tilde{\alpha}_{M U}$, where $\tilde{\alpha}_{M U}$ is as described in Perron and Yabu (2009), who show this truncation gives the better finite sample power properties. The $t_{\beta}^{R Q F}$ statistic is asymptotically standard normal under the null hypothesis when $u_{t}$ is either $\mathrm{I}(0)$ or exact I(1) and, as noted in Remark 2 of Perron and Yabu (2009), has the same local asymptotic power as the $z_{\lambda}$ statistic of Harvey et al. (2007) in the local-to-unity autoregressive root environment that we consider in this paper.

\section{Modified Tests}

Our proposed testing procedure involves utilising similar $t$-ratios to Vogelsang (1998) and Bunzel and Vogelsang (2005), although we propose use of the auxiliary $J$ unit root test to switch between the exact $\mathrm{I}(1)$ and $\mathrm{I}(0)$ critical values. Such an approach has the advantage that it leads to a test with local asymptotic power identical to the tests of Vogelsang (1998) and Bunzel and Vogelsang (2005) when the errors are $\mathrm{I}(0)$, but with greater local asymptotic power when the shocks are $\mathrm{I}(1)$ due to the removal of the influence of the auxiliary unit root test statistic on the asymptotic distribution of the test in the $\mathrm{I}(1)$ case. Specifically, the first test statistic is a modified version of the $t P S W^{1}$ test of Vogelsang (1998) calculated as

$$
t P S W_{s}^{1}:=\frac{\hat{\beta}-\beta_{0}}{\sqrt{T^{-1} 100 s_{z}^{2}}} .
$$


Notice that this is identical to the original $t P S W^{1}$ statistic of Vogelsang (1998) but excluding the scaling factor $\exp \left(-c_{\xi} J\right)$. We then calculate the weight function

$$
\lambda^{\prime}:=\exp \left(-\tau T^{1 / 2} J^{v}\right)
$$

with constants $v>0.5$ and $\tau>0$, and compare the $t P S W_{s}^{1}$ test statistic to a critical value, $c v$, given by

$$
c v:=\left(1-\lambda^{\prime}\right) c v_{I(1)}+\lambda^{\prime} c v_{I(0)}
$$

where $c v_{I(1)}$ and $c v_{I(0)}$ are the asymptotic critical values of the $t P S W_{s}^{1}$ statistic at the desired significance level in the exact $\mathrm{I}(1)$ and $\mathrm{I}(0)$ environments, respectively. The rationale behind such an approach is that when the error process, $u_{t}$, is $\mathrm{I}(1), J^{v}$ is $O_{p}(1)$, so that $T^{1 / 2} J^{v}$ is $O_{p}\left(T^{1 / 2}\right)$ and hence $\lambda^{\prime} \stackrel{p}{\rightarrow} 0$ and so the exact $\mathrm{I}(1)$ critical value is used. Conversely, when $u_{t}$ is $\mathrm{I}(0), J^{v}$ is $O_{p}\left(T^{-v}\right)$, so that $T^{1 / 2} J^{v}$ is $o_{p}(1)$ given $v>0.5$, and now $\lambda^{\prime} \stackrel{p}{\rightarrow} 1$ so that the $\mathrm{I}(0)$ critical value is used. Consequently, at least asymptotically, the test will have correct size regardless of whether the error process is $\mathrm{I}(0)$ or exact $\mathrm{I}(1)$. This holds irrespective of the values of $\tau>0$ and $v>0.5$, which are calibrated later in the paper to best control the size of the test in finite samples. ${ }^{1}$

We also apply the same principle to the test statistic of Bunzel and Vogelsang (2005), with our proposed test statistic given by

$$
{\operatorname{Dan}-J_{s}}_{s}:=\frac{\hat{\beta}-\beta_{0}}{\sqrt{\hat{\omega}_{u}^{2} / \sum_{t=1}^{T}(t-\bar{t})^{2}}} .
$$

We then calculate the scaling factor $\lambda^{\prime}$ in the same way as previously described and use this to switch between the relevant critical values in the exact $\mathrm{I}(1)$ and $\mathrm{I}(0)$ environments. With this procedure the critical values will, as with the test of Bunzel and Vogelsang (2005), depend on the choice of bandwidth, $b$, used to estimate the long run variance.

\section{Asymptotic Results}

We now examine the asymptotic behaviour of the test statistics outlined in this paper. We consider the size and power properties of the test under both the null hypothesis $H_{0}: \beta=\beta_{0}$ and the local alternative hypotheses $H_{10}: \beta=\beta_{0}+\kappa T^{-3 / 2}$ and $H_{11}: \beta=\beta_{0}+\kappa T^{-1 / 2}$, where $\kappa$ is a finite constant, with the $T$-scalings providing the appropriate Pitman drift under $\mathrm{I}(0)$ and $\mathrm{I}(1)$ errors, respectively. The limiting distributions are expressed in terms of the following functions defined below.

\footnotetext{
${ }^{1}$ Notice that the required properties for the large sample behaviour of $\lambda^{\prime}$ under $\mathrm{I}(0)$ and $\mathrm{I}(1)$ errors are also satisfied by $1-\lambda^{*}$, where $\lambda^{*}$ is the weight function (5) used by Harvey et al. (2007). Other specifications for $\lambda^{\prime}$ could also be used, provided that $\lambda^{\prime} \stackrel{p}{\rightarrow} 0$ under $\mathrm{I}(1)$ and $\lambda^{\prime} \stackrel{p}{\rightarrow} 1$ under $\mathrm{I}(0)$. The weight function adopted in (6) has the advantage of requiring the computation of only one auxiliary statistic. Moreover, while less parameterized specifications could be adopted, we found that the greater flexibility permitted by the inclusion of $v$ and $\tau$ delivered improved overall finite sample performance.
} 


\section{Definition 1}

$$
\begin{aligned}
& F(r):=[1, r]^{\prime}, \quad G(r):=\left[r,(1 / 2) r^{2}\right]^{\prime}, \quad Q(r):=\left[1, r, r^{2}, \ldots, r^{9}\right]^{\prime}, \quad R^{*}:=[0,1] \\
& N^{F}:= \begin{cases}\int_{0}^{1} F(s) d w(s) & \text { if }|\alpha|<1 \\
\int_{0}^{1} F(s) w_{\bar{\alpha}}(s) d s & \text { if } \alpha=1-\bar{\alpha} / T\end{cases} \\
& H(r):= \begin{cases}w(r) & \text { if }|\alpha|<1 \\
\int_{0}^{r} w_{\bar{\alpha}}(s) d s & \text { if } \alpha=1-\bar{\alpha} / T\end{cases} \\
& Q^{F}(r):=H(r)-\int_{0}^{r} F(s)^{\prime} d s\left(\int_{0}^{1} F(s) F(s)^{\prime} d s\right)^{-1} N^{F} \\
& \Phi^{F}(b, k):=\int_{0}^{1} \int_{0}^{1}-k^{\prime \prime}((r-s) / b) Q^{F}(r) Q^{F}(s) d r d s \\
& A_{c}:=\frac{\int_{0}^{1} L_{c}\left(w_{\bar{\alpha}}(r), F(r)\right)^{2} d r}{\int_{0}^{1} L_{c}\left(w_{\bar{\alpha}}(r), Q(r)\right)^{2} d r}-1,
\end{aligned}
$$

where $k^{\prime \prime}(x)$ is the second derivative of the Bartlett kernel and $L_{c}(p, q)$ generically denotes the continuous time residuals from the projection of $p$ onto the space spanned by $q$.

Consider first the $\mathrm{I}(0)$ case. To that end if $y_{t}$ is generated according to (1) and (2) with $|\alpha|<1$ and $\beta=\beta_{0}+\kappa T^{-3 / 2}$, then,

$$
\begin{aligned}
t P S W^{1}, t P S W_{s}^{1} & \stackrel{d}{\rightarrow} \frac{\kappa / \sigma+R^{*^{\prime}}\left(\int_{0}^{1} F(s) F(s)^{\prime} d s\right)^{-1} N^{F}}{\sqrt{100 \int_{0}^{1} L_{c}(w(r), G(r))^{2} d r R^{*}\left(\int_{0}^{1} F(s) F(s)^{\prime} d s\right)^{-1} R^{*^{\prime}}}} \\
z_{\lambda}, z_{\lambda}^{m 2}, t_{\beta}^{R Q F} & \stackrel{d}{\rightarrow} w(1)+\kappa /(\sqrt{12} \sigma) \\
\text { Dan-J, Dan- } J_{s} & \stackrel{d}{\rightarrow} \frac{\kappa / \sigma+R^{*^{\prime}}\left(\int_{0}^{1} F(s) F(s)^{\prime} d s\right)^{-1} N^{F}}{\sqrt{\Phi^{F}(b, k) R^{*}\left(\int_{0}^{1} F(s) F(s)^{\prime} d s\right)^{-1} R^{*^{\prime}}}}
\end{aligned}
$$

The proofs for the $t P S W_{s}^{1}$ and $D a n-J_{s}$ statistics follow trivially from those of the $t P S W^{1}$ and Dan-J statistics given in Vogelsang (1998) and Bunzel and Vogelsang (2005). The proofs for the $z_{\lambda}$, $z_{\lambda}^{m 2}$ and $t_{\beta}^{R Q F}$ tests are taken from Harvey et al. (2007) and Perron and Yabu (2009). The modified and unmodified versions of the statistics of Vogelsang (1998) and Bunzel and Vogelsang (2005) share the same asymptotic distribution in the $\mathrm{I}(0)$ case and, as such, will have the same asymptotic local power for any given $\kappa$ when $u_{t}$ is $\mathrm{I}(0)$. This is due to the fact that the scaled unit root statistic in the original test statistics converges to 1 , and the critical value utilised for the modified statistic converges to the $\mathrm{I}(0)$ critical value, yielding identical tests in the limit. 
Next consider the I(1) case. If $y_{t}$ is generated according to (1) and (2) with $\alpha=1-\bar{\alpha} / T$ and $\beta=\beta_{0}+\kappa T^{-1 / 2}$, then

$$
\begin{aligned}
& t P S W^{1} \stackrel{d}{\rightarrow} \frac{\kappa / \omega_{\varepsilon}+R^{*^{\prime}}\left(\int_{0}^{1} F(s) F(s)^{\prime} d s\right)^{-1} N^{F}}{\sqrt{100 \int_{0}^{1} L_{c}\left(\int_{0}^{1} w_{\bar{\alpha}}(s) d s, G(r)\right)^{2} d r R^{*}\left(\int_{0}^{1} F(s) F(s)^{\prime} d s\right)^{-1} R^{*^{\prime}}}} \operatorname{xp}\left(-c_{\xi} A_{c}\right) \\
& \text { Dan-J } \stackrel{d}{\rightarrow} \frac{\kappa / \sigma+R^{*^{\prime}}\left(\int_{0}^{1} F(s) F(s)^{\prime} d s\right)^{-1} N^{F}}{\sqrt{\Phi^{F}(b, k) R^{*}\left(\int_{0}^{1} F(s) F(s)^{\prime} d s\right)^{-1} R^{*^{\prime}}}} \exp \left(-c_{\xi} A_{c}\right) \\
& z_{\lambda}, t_{\beta}^{R Q F} \stackrel{d}{\rightarrow} w_{\bar{\alpha}}(1)+\kappa / \omega_{\varepsilon} \\
& z_{\lambda}^{m 2} \stackrel{d}{\rightarrow} \delta_{\gamma}\left(\int_{0}^{1} L_{c}\left(w_{\bar{\alpha}}(r), F(r)\right) d r\right)^{-2}\left(w_{\bar{\alpha}}(1)+\kappa / \omega_{\varepsilon}\right) \\
& t P S W_{s}^{1} \stackrel{d}{\rightarrow} \frac{\kappa / \omega_{\varepsilon}+R^{*^{\prime}}\left(\int_{0}^{1} F(s) F(s)^{\prime} d s\right)^{-1} N^{F}}{\sqrt{100 \int_{0}^{1} L_{c}\left(\int_{0}^{1} w_{\bar{\alpha}}(s) d s, G(r)\right)^{2} d r R^{*}\left(\int_{0}^{1} F(s) F(s)^{\prime} d s\right)^{-1} R^{*^{\prime}}}} \\
& \operatorname{Dan}_{-} J_{s} \stackrel{d}{\rightarrow} \frac{\kappa / \sigma+R^{*^{\prime}}\left(\int_{0}^{1} F(s) F(s)^{\prime} d s\right)^{-1} N^{F}}{\sqrt{\Phi^{F}(b, k) R^{*}\left(\int_{0}^{1} F(s) F(s)^{\prime} d s\right)^{-1} R^{*^{\prime}}}}
\end{aligned}
$$

The proofs for the $t P S W_{s}^{1}$ and Dan- $J_{s}$ statistics, again, follow trivially from those of the $t P S W^{1}$ and Dan-J statistics given in Vogelsang (1998) and Bunzel and Vogelsang (2005). The proofs for the $z_{\lambda}, z_{\lambda}^{m 2}$ and $t_{\beta}^{R Q F}$ tests are, once again, taken from Harvey et al. (2007) and Perron and Yabu (2009). As can be seen, the limiting distributions of the modified versions of the $t P S W_{s}^{1}$ and $D a n-J_{s}$ statistics now differ from those of the original versions of these statistics. This is due to the fact that the $J$ unit root test impacts the asymptotic distribution of the original statistics, which will in turn affect their local asymptotic power, whereas for the modified tests this same test statistic is simply used to select the critical value, which will have no effect on local asymptotic power. The main difference between the modified and unmodified statistics is that the additional variation from the $J$ unit root test statistic impacts the asymptotic distribution of the $t P S W^{1}$ and Dan-J statistics, whereas for the $t P S W_{s}^{1}$ and $D a n-J_{s}$ statistics this variation impacts the critical value selected asymptotically. In the exact $\mathrm{I}(1)$ case we will show that this leads to large power gains for the modified tests relative to their unmodified counterparts.

We now examine the asymptotic power functions of all of the above tests by directly simulating the limiting representations given above. The Weiner processes were approximated using NIID $(0,1)$ random variates and approximating integrals by normalised sums of 1000 steps. All Monte Carlo simulations that follow were performed in Gauss 9.0 using 10,000 replications. The results we report are for one-sided tests of $H_{0}: \beta=\beta_{0}$ against $H_{1}^{\prime}: \beta>\beta_{0}$. Consequently, results are reported for positive values of $\kappa$ only. Notice, however, that these power functions are identical to those of the corresponding one-sided tests of $H_{0}: \beta=\beta_{0}$ against $H_{1}^{\prime} \hat{a} e^{\mathrm{TM}}: \beta<\beta_{0}$ which obtain for negative values 
of $\kappa$. All results are presented at an asymptotic level of $5 \%$. For the Dan- $J_{s}$ test a bandwidth of $b=0.02$ was utilised in all scenarios as this choice of bandwidth led to a test with the greatest local asymptotic power for both $\mathrm{I}(1)$ and $\mathrm{I}(0)$ errors, as such, we recommend the use of this bandwidth when performing the Dan- $J_{s}$ test in practice and perform the test utilising this bandwidth for the remainder of the paper. For the Dan-J test results are reported for $b=b_{\text {opt }}(\bar{\alpha})$. Note that all tests are constructed to give an asymptotic size of $5 \%$ in the $\mathrm{I}(0)$ and exact $\mathrm{I}(1)$ environment, thus for the case of errors that are near I(1) with $\bar{\alpha}>0$ the tests will be conservative.

For the case of $\mathrm{I}(0)$ errors results are reported for the null hypothesis $H_{0}: \beta=\beta_{0}$ and the local alternative $H_{10}: \beta=\beta_{0}+\kappa T^{-3 / 2}$. We consider a range of $\kappa \in[0,20]$ using a grid with 100 steps, with results reported in Figure 1. As can be seen, the greatest power is achieved by the $z_{\lambda}, z_{\lambda}^{m 2}$ and $t_{\beta}^{R Q F}$ tests, with all of these tests attaining the Gaussian asymptotic local power envelope. The Dan-J and $D a n-J_{s}$ tests have identical asymptotic local power functions, as do the $t P S W^{1}$ and $t P S W_{s}^{1}$ tests. Although the tests based on Dan- $J$ have uniformly better power than the tests based on $t P S W^{1}$, they are slightly less powerful than the $z_{\lambda}, z_{\lambda}^{m 2}$ and $t_{\beta}^{R Q F}$ tests which are asymptotically optimal in the $\mathrm{I}(0)$ environment.

For the case of near I(1) errors results are reported for the null hypothesis $H_{0}: \beta=\beta_{0}$ and the local alternative $H_{11}: \beta=\beta_{0}+\kappa T^{-1 / 2}$ for a range of $\bar{\alpha} \in[0,5,10,15]$, with $\bar{\alpha}=0$ corresponding to an exact $\mathrm{I}(1)$ process. We consider a range of $\kappa \in[0,8]$ using a grid with 100 steps. Figure 2(a) shows a clear ordering in the asymptotic local power of the test statistics in the exact I(1) case, with the $z_{\lambda}$ and $t_{\beta}^{R Q F}$ tests achieving the Gaussian asymptotic local power envelope and displaying the greatest overall power. The Dan- $J_{s}$ and $t P S W_{s}^{1}$ tests display power significantly in excess of their unmodified counterparts, with the $z_{\lambda}^{m 2}$ test attaining power somewhere between the modified and unmodified tests. The superiority of the new modified tests, compared with their unmodified counterparts, is unsurprising as the choice to utilise the auxiliary unit root test statistic to select the critical value rather than scale the test statistic itself removes the influence of the auxiliary unit root test statistic on the asymptotic distribution of the test in the $\mathrm{I}(1)$ case, leading to greater local asymptotic power.

The results are more mixed when $\bar{\alpha}>0$, with no single test having uniformly greater power for all values of $\kappa$. Results for $\bar{\alpha}=5$, reported in Figure 2(b), show that all of the procedures are under-sized, particularly so in the case of the $z_{\lambda}$ and $t_{\beta}^{R Q F}$ tests. In this scenario it is, in fact, the original $t P S W^{1}$ and Dan-J tests that have the best power properties for values of $\kappa$ less than around 1.6, followed by the $z_{\lambda}^{m 2}, t P S W_{s}^{1}$ and Dan-Js tests, with the $z_{\lambda}$ and $t_{\beta}^{R Q F}$ tests having the lowest overall power. For values of $\kappa$ greater than around 1.6, however, the power ordering is reversed, with the tests reverting to the power ordering observed for the exact I(1) case. In particular the $z_{\lambda}, t_{\beta}^{R Q F}$, Dan-J $J_{s}$ and $t P S W_{s}^{1}$ tests display power significantly in excess of the $z_{\lambda}^{m 2}, t P S W^{1}$ and Dan- $J$ tests for moderate values of $\kappa$.

The pattern of results for $\bar{\alpha}=10$ and $\bar{\alpha}=15$, presented in Figures 2(c) and 2(d), respectively, are broadly similar to the case of $\bar{\alpha}=5$, although as $\bar{\alpha}$ increases the $z_{\lambda}^{m 2}$ test begins to dominate other tests for values of $\kappa$ less than around 1.6. Whilst the power ordering is otherwise identical, the difference in power between the $z_{\lambda}^{m 2}, t P S W^{1}$ and Dan-J tests and all other tests for $\kappa$ less than 
around 1.6 is increasing in $\bar{\alpha}$, with the power functions of the $z_{\lambda}$ and $t_{\beta}^{R Q F}$ tests converging towards a step function at $\kappa=1.645$ as $\bar{\alpha}$ is increased.

In summary, if we are interested in only $\mathrm{I}(0)$ or exact $\mathrm{I}(1)$ processes then the $z_{\lambda}$ and $t_{\beta}^{R Q F}$ tests have uniformly greater local asymptotic power. The results are, however, mixed if we allow for the case of near I(1) processes. The most important point to note, however, is that in the exact I(1) scenario using the $J$ unit root test statistic to select a critical value in the testing procedure of Vogelsang (1998) and Bunzel and Vogelsang (2005) rather than scaling the test statistic itself yields substantial power gains, with the $t P S W_{s}^{1}$ and $D a n-J_{s}$ tests displaying power far in excess of the original $t P S W^{1}$ and Dan-J tests. For near I(1) processes results are less clear, with the modified versions of the test of Vogelsang (1998) and Bunzel and Vogelsang (2005) displaying power below their unmodified counterparts for small values of $\kappa$, but displaying greater power for larger values of $\kappa$. It will, therefore, be important to examine how this pattern of local asymptotic power translates into the finite sample performance of the proposed tests.

\section{Finite Sample Simulations}

In this section we present the results from a Monte Carlo simulation exercise performed to assess the finite sample size and power properties of the tests discussed in this paper. Data were generated according to

$$
\begin{aligned}
& y_{t}=\mu+\beta t+u_{t}, \quad t=1, \ldots, T, \\
& u_{t}=\alpha_{T} u_{t-1}+e_{t}+\theta e_{t-1},
\end{aligned}
$$

with $e_{t} \sim \operatorname{NIID}(0,1), \alpha_{T}=1-\bar{\alpha} / T$ and $u_{1}=e_{1}=e_{0}=0$. We test the null hypothesis $H_{0}: \beta=\beta_{0}$ against the one-sided alternative $H_{1}^{\prime}: \beta>\beta_{0}$, using one-sided tests, and where without loss of generality we set $\beta_{0}=\mu=0$. All tests were performed at the nominal $5 \%$ level. When performing the $t P S W_{s}^{1}$ and Dan-J $J_{s}$ tests values of $\tau$ and $v$ used when calculating the relevant critical value were calibrated to give the best overall small sample performance. We considered values of $v=1$ and $v=2$ and for each test and value of $v$ a value of $\tau$ was chosen such that size was controlled across all scenarios considered. For a given $v$ smaller values of $\tau$ lead to a test with higher finite sample size due to more weight being placed on the $\mathrm{I}(0)$ critical value, whereas larger values of $\tau$ lead to lower finite sample size as more weight is placed on the $\mathrm{I}(1)$ critical value. We found that $v=2$ for both tests and values of $\tau=0.03$ and $\tau=0.074$ for the $t P S W_{s}^{1}$ and Dan- $J_{s}$ tests, respectively, led to tests with well controlled size for i.i.d errors in all scenarios and decent finite sample power properties.

Table 1 reports the size of the testing procedures for a range of $\bar{\alpha} \in[0,5,10,15]$ and $\theta \in$ $[-0.8,-0.4,0.0,0.4,0.8]$. We see that all the tests except the $t P S W^{1}$ and Dan-J tests have poor size control when $\bar{\alpha}=0, T=100$ and the errors are i.i.d., with the $z_{\lambda}$ test displaying the worst overall size distortions with an empirical size of $11.9 \%$. The $t P S W_{s}^{1}$ and Dan-J tests are also oversized, but not to such an extent. These size distortions are reduced when the sample size is increased to 
$T=250$ but are still noticeable for all but the $t P S W^{1}$ and Dan- $J$ tests. For the near integrated scenarios considered, all of the tests are conservative when the errors are i.i.d., with actual size below the $5 \%$ nominal level for all values of $\bar{\alpha}>0$. Moving away from the i.i.d. case, we see that introducing negative MA behaviour into the noise function leads to an increase in the size of all tests for all values of $\bar{\alpha}$, with the exception of the $z_{\lambda}$ and $z_{\lambda}^{m 2}$ tests. For these two tests size is relatively unaffected by the value of $\theta$, with only the case $\theta<0$ and $\bar{\alpha}=0$ leading to a reduction in size. For the remaining tests, size distortions are most noticeable for the tests based on Dan-J, although the tests based on $t P S W^{1}$ do suffer quite severe size distortions when $\bar{\alpha}=0$ and $\theta<0$. The size of all tests are less sensitive to positive MA behaviour, with the size of the tests in this scenario almost identical to the i.i.d. case.

We now turn our attention to the power of the tests under the alternative. Figure 3 reports results for $\bar{\alpha}=0$ for sample sizes of $T=100$ and $T=250$ and values of $\theta \in[-0.4,0.0,0.4]$. In this scenario there is a clear ordering in the power of the tests, with $z_{\lambda}$ having uniformly greater power than all tests, closely followed by $t_{\beta}^{R Q F}$. The $t P S W_{s}^{1}$ and $D a n-J_{s}$ tests have power significantly in excess of their unmodified counterparts, with power of the $z_{\lambda}^{m 2}$ test somewhere between that of the $t P S W_{s}^{1}$ and the Dan-J test. These results are unsurprising given that the most powerful tests in this scenario are also those that are most oversized. As such, Figure 4 reports size adjusted power for the same scenarios. For a sample size of $T=100$ with i.i.d. errors the best size adjusted power is given by the $t_{\beta}^{R Q F}, z_{\lambda}$ and Dan-J $J_{s}$ tests, with the $t P S W_{s}^{1}$ test the most powerful of the remaining tests. When the sample size is increased to $T=250$ the results are fairly similar, although the $t_{\beta}^{R Q F}$ test now shows the best power overall, followed by $z_{\lambda}$, then $D a n-J_{s}$. We see a similar pattern of results when we allow for positive MA behaviour in the error process, although when we allow for negative behaviour when $\theta=-0.4$ the results are slightly different. In this scenario the best overall power is now achieved by the $z_{\lambda}$ and Dan- $J_{s}$ tests, followed by the $t P S W_{s}^{1}$ and $t_{\beta}^{R Q F}$ tests.

Figure 5 reports the power of the tests for $\bar{\alpha}=5$. In this scenario results are mixed, with the $z_{\lambda}$ test displaying arguably the best overall power, although it is somewhat less powerful than the other tests for smaller values of $\beta$, reflecting the local asymptotic power results in Figure 2(b). The most important results to note in this scenario are that, much like in the case of exact I(1) errors, the $t P S W_{s}^{1}$ and $D a n-J_{s}$ tests have better overall power than their unmodified counterparts. The power of the $t P S W_{s}^{1}$ test is uniformly higher than that of the $t P S W^{1}$ test, and the Dan-J $J_{s}$ test is more powerful than the Dan-J test for all but very small values of $\beta$. The $t P S W_{s}^{1}$ test also shows higher power than both the $z_{\lambda}$ and $t_{\beta}^{R Q F}$ tests for lower values of $\beta$.

Figures 6 and 7 report the power of the tests for $\bar{\alpha}=10$ and $\bar{\alpha}=15$, respectively. Results here are, once again, rather mixed. The power functions of the tests are much closer together, particularly for $\bar{\alpha}=15$, with no one test dominating the others. What is important to note, however, is that the $t P S W_{s}^{1}$ test, once again, shows uniformly greater power than its unmodified counterpart. The Dan- $J_{s}$ test, however, does not perform so well with the power of this test falling below that of the original $D a n-J$ test for all but large values of $\beta$. The $t P S W_{s}^{1}$ test is again competitive with the $z_{\lambda}$ and $t_{\beta}^{R Q F}$ tests.

The results reported in this section suggest that no one test is best suited to the testing problem 
at hand. The important result is that the $t P S W_{s}^{1}$ test shows uniformly greater finite sample power than the original $t P S W^{1}$ test, and often has the best power properties of all tests for smaller values of $\beta$ and when $\bar{\alpha}>0$. Results for the $\operatorname{Dan}_{-} J_{s}$ test are less clear cut. While the power of this test is greater than both the $t P S W_{s}^{1}$ and Dan-J tests in the exact I(1) environment, it appears to show inferior power properties to these two tests in the near integrated environment.

\section{Application to Equity Indices}

We now apply the test statistics outlined in this paper to a number of stock market indices. We consider the natural logarithm of the monthly closing price of six equity indices using all available data sourced from Yahoo! finance. The six indices utilised are the NASDAQ (02/1971-10/2013), S\&P100 (08/1982-10/2013), S\&P500 (01/1950-10/2013), FTSE 100 (04/1984-10/2013), FTSE 250 (12/1985-10/2013) and the FTSE All Share (12/1972-10/2013). For each index, Table 2 reports whether one-sided implementations of the tests outlined in this paper reject in favour of a positive trend at the $10 \%, 5 \%$ or $1 \%$ significance levels. We also report the associated estimate of $\alpha_{T}$ obtained from OLS estimation of (1)-(2), denoted $\hat{\alpha}$. Figure 8 plots the series and the fitted deterministic components, whilst Figure 9 plots the detrended series.

The rejection patterns associated with the different tests appear to mirror the asymptotic and

finite sample power results reported in Sections 5 and 6 . The $z_{\lambda}$ and $t_{\beta}^{R Q F}$ tests detect a linear trend for each of the six equity indices, with the $t P S W_{s}^{1}$ and $D a n-J_{s}$ tests indicating trends in four and five, respectively, of the six series. We note that while the $t P S W_{s}^{1}$ and Dan- $J_{s}$ tests fail to detect a trend in some cases they do, however, provide more evidence for the presence of a deterministic trend than their unmodified counterparts $t P S W^{1}$ and Dan-J; moreover, they never fail to detect a trend where it is detected by the unmodified tests.

For each series, at least one of the tests indicates the presence of a deterministic linear trend, lending strong support to the notion that equity price indices are subject to (positive) long run growth. This implies non-zero long run average returns, and therefore an investment strategy of buy and hold for an index would be expected to deliver positive returns equal to the long run growth rate. An obvious implication of these findings is that any subsequent tests of the EMH should take account of a long run trend component in prices (or a non-zero mean component in returns).

Examining the values of $\hat{\alpha}$ in Table 2 it is seen that the residuals from the detrended series are, in all instances, compatible with processes which are either $\mathrm{I}(1)$ or $\mathrm{I}(0)$ but highly persistent. As a further indication as to the integration properties of the data, the with-trend local GLS unit root test statistic of Elliott et al. (1996), DF-GLS ${ }^{\tau}$, is also reported for each series in Table 2. We see from these results that the unit root null is not rejected at conventional significance levels for five of the six series; for the FTSE 250 index, a rejection is found at the $10 \%$ significance level. That there is some modest evidence of stationary, as well as unit root, behaviour across the different series reinforces the importance of using procedures for trend detection that are robust to the order of integration of the data being analysed. Given that the procedures considered in this paper achieve such robustness 
asymptotically, we may conclude that deterministic trends are present in these stock indices with a reasonable degree of confidence, without needing to explicitly model the stochastic component of the series.

\section{Conclusions}

In this paper we have proposed a modification to the trend tests of Vogelsang (1998) and Bunzel and Vogelsang (2005) in which a unit root test statistic is used to select the critical value utilised in the trend test procedure rather than being used to scale the test statistic itself, the latter being the approach in Vogelsang (1998) and Bunzel and Vogelsang (2005). We have shown that these modified tests have uniformly greater local asymptotic power than their unmodified counterparts in the exact $\mathrm{I}(1)$ environment and identical local asymptotic power in the $\mathrm{I}(0)$ environment, and that the modified version of the test of Vogelsang (1998) not only has uniformly greater finite sample power than its unmodified counterpart, but also has power in the near I(1) environment that is competitive with the asymptotically optimal tests of Harvey et al. (2007) and Perron and Yabu (2009). That this modified test is able to dominate the test of Vogelsang (1998) across most scenarios whilst attaining power which is competitive with tests designed to be optimal in the exact $\mathrm{I}(1)$ and $\mathrm{I}(0)$ environment is encouraging, and motivates the use of this modification in other testing problems where the test statistic has a different limiting distribution in the $\mathrm{I}(1)$ and $\mathrm{I}(0)$ environments. In the current context of testing for a linear trend, applying the tests examined in this paper to a number of equity indices uncovers strong support for the presence of deterministic trends in the series, implying that long run growth represents an important characteristic of such stock price indices.

\section{References}

Bunzel, H. and Vogelsang, T.J. (2005). Powerful trend function tests that are robust to strong serial correlation, with an application to the Prebisch-Singer hypothesis. Journal of Business and Economic Statistics 23, 381-94.

Elliott, G., Rothenberg, T.J. and Stock, J.H. (1996). Efficient tests for an autoregressive unit root. Econometrica 64, 813-36.

Harvey, D.I., Leybourne, S.J., and Taylor, A.M.R. (2007). A simple, robust and powerful test of the trend hypothesis. Journal of Econometrics 141, 1302-30.

Jansson, M. (2002). Consistent covariance matrix estimation for linear processes. Econometric Theory 18, 1449-1459.

Kwiatkowski, D., Phillips, P.C.B., Schmidt, P. and Shin, Y. (1992). Testing the null hypothesis of stationarity against the alternative of a unit root: how sure are we that economic time series have a unit root? Journal of Econometrics 54, 159-78. 
Kiefer, N. M. and Vogelsang, T. J. (2005). A new asymptotic theory for heteroskedasticity-autocorrelation robust tests. Econometric Theory 21, 1130-1164.

Marsh, P.W.N. (2009). The properties of Kullback-Leibler divergence for the unit root hypothesis. Econometric Theory 25, 1662-81.

Park, J.Y. (1990). Testing for unit roots and cointegration by variable addition, in Fomby, T. and Rhodes, F. (eds.) Advances in Econometrics: Cointegration, Spurious Regression and Unit Roots. Jai Press: Greenwich.

Park, J.Y. and Choi, B. (1988). A new approach to testing for a unit root. CAE Working Paper 88-23, Cornell University.

Perron, P. (1998). Trends and random walks in macroeconomic time series: further evidence from a new approach. Journal of Economic Dynamics and Control 12, 297-332.

Perron, P. and Yabu, T. (2009). Estimating deterministic trends with an integrated or stationary noise component. Journal of Econometrics 151, 56-69.

Roy, A., Falk, B. and Fuller, W.A. (2004). Testing for trend in the presence of autoregressive errors. Journal of the American Statistical Association 99, 1082-1091.

Roy, A. and Fuller, W.A. (2001). Estimation for autoregressive processes with a root near one. Journal of Business and Economic Statistics 19, 482-493.

Vogelsang, T.J. (1998). Trend function hypothesis testing in the presence of serial correlation. Econometrica $66,123-148$. 


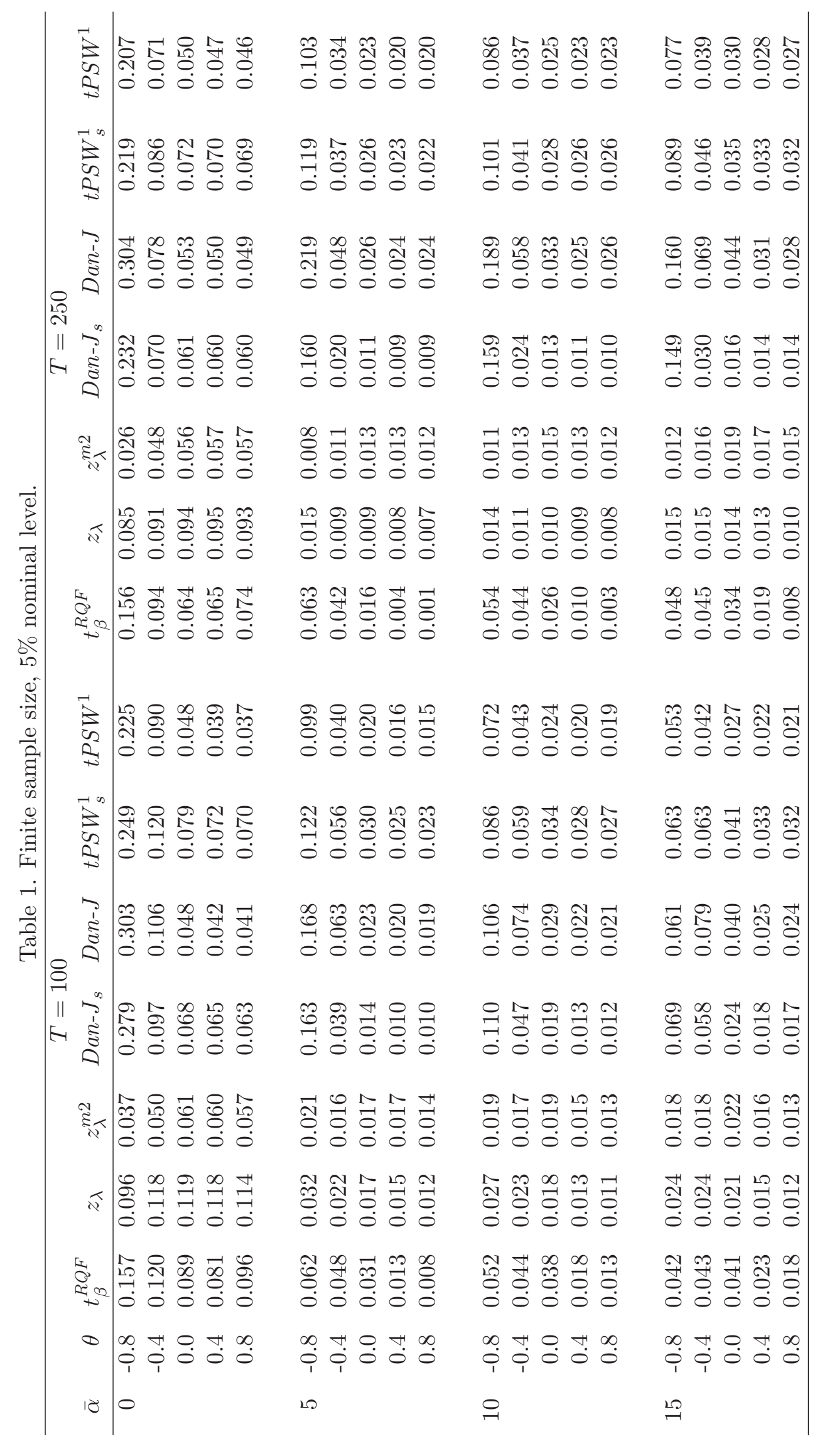


Table 2. Results of Tests Applied to Equity Indices

\begin{tabular}{ccccccc}
\hline & NASDAQ & SP100 & SP500 & FTSE 100 & FTSE 250 & FTSE AS \\
\hline$t P S W^{1}$ & $* *$ & - & - & - & $* * *$ & - \\
$t P S W_{s}^{1}$ & $* *$ & - & $* *$ & - & $* * *$ & $*$ \\
Dan- $J$ & $*$ & - & $*$ & - & $* * *$ & - \\
Dan- $J_{s}$ & $* * *$ & - & $* * *$ & $*$ & $* * *$ & $* *$ \\
$z_{\lambda}$ & $* * *$ & $* *$ & $* * *$ & $* *$ & $* * *$ & $* *$ \\
$z_{\lambda}^{m 2}$ & $* *$ & - & $*$ & - & $* * *$ & - \\
$t_{\beta}^{R Q F}$ & $* *$ & $* *$ & $* * *$ & $* *$ & $* * *$ & $* *$ \\
$\hat{\alpha}$ & 0.98 & 0.97 & 0.99 & 0.98 & 0.94 & 0.99 \\
DF-GLS $^{\tau}$ & -2.28 & -1.30 & -2.03 & -1.29 & $-2.65 *$ & -1.62 \\
\hline
\end{tabular}

Test Rejected at ${ }^{*} 10 \%$ level, ${ }^{* *} 5 \%$ level, ${ }^{* * *} 1 \%$ level 


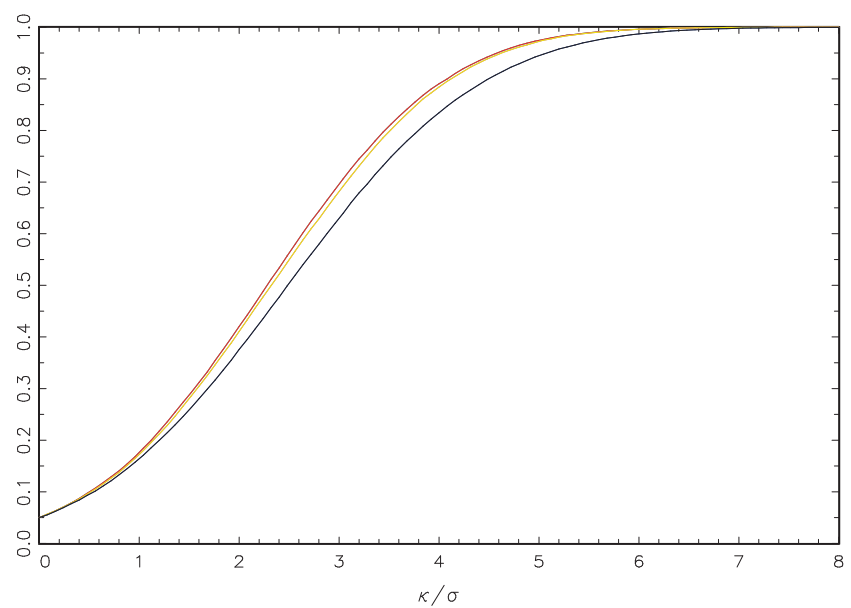

$z_{\lambda}, t_{\beta}^{R Q F}, z_{\lambda}^{m 2}:-$ Dan- $_{s}$, Dan-J: $\quad, t P S W_{s}^{1}, t P S W^{1}$

Figure 1. Local Asymptotic Power: I(0) innovations

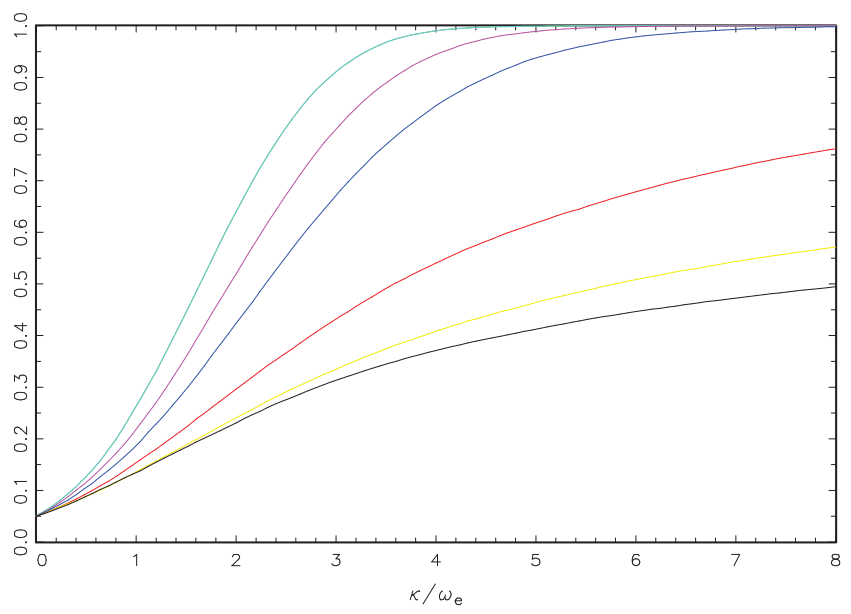

(a) $\bar{\alpha}=0$

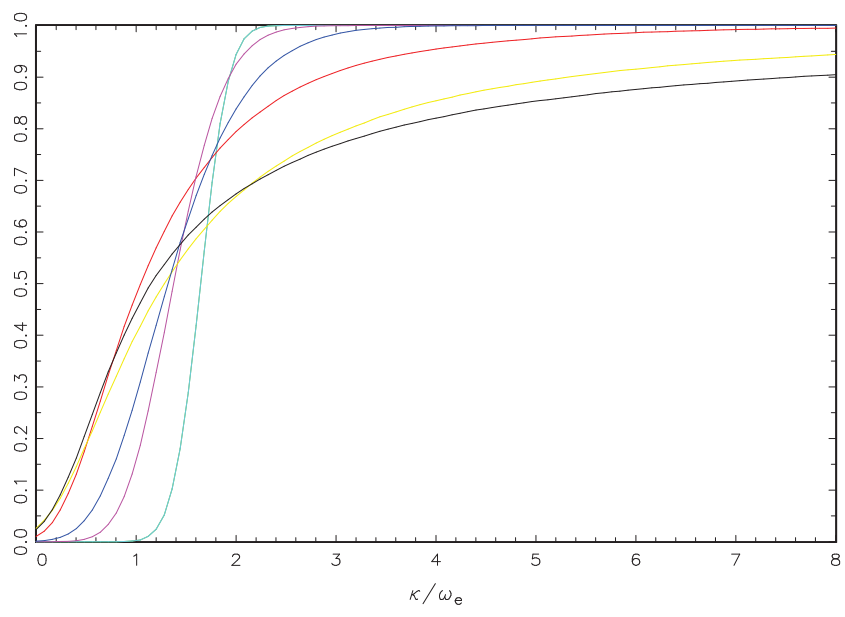

(a) $\bar{\alpha}=10$

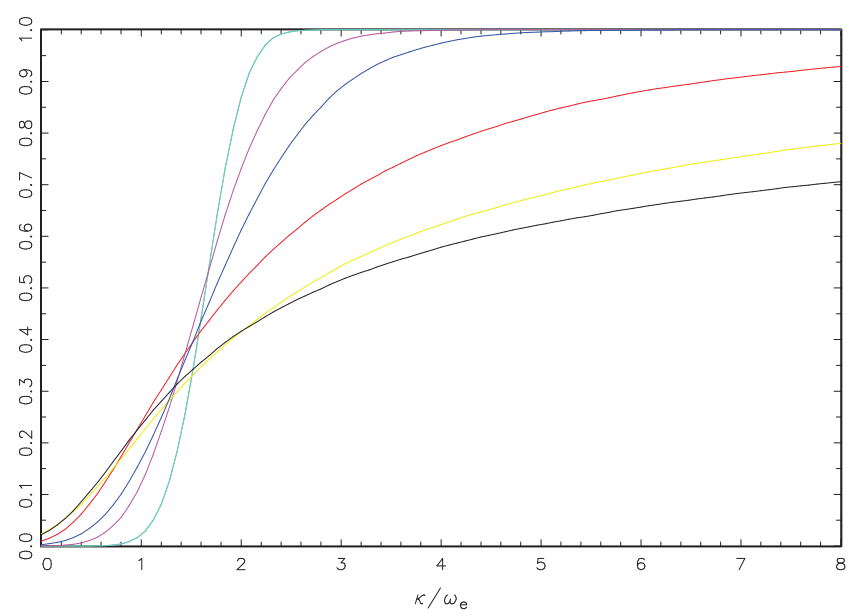

(b) $\bar{\alpha}=5$

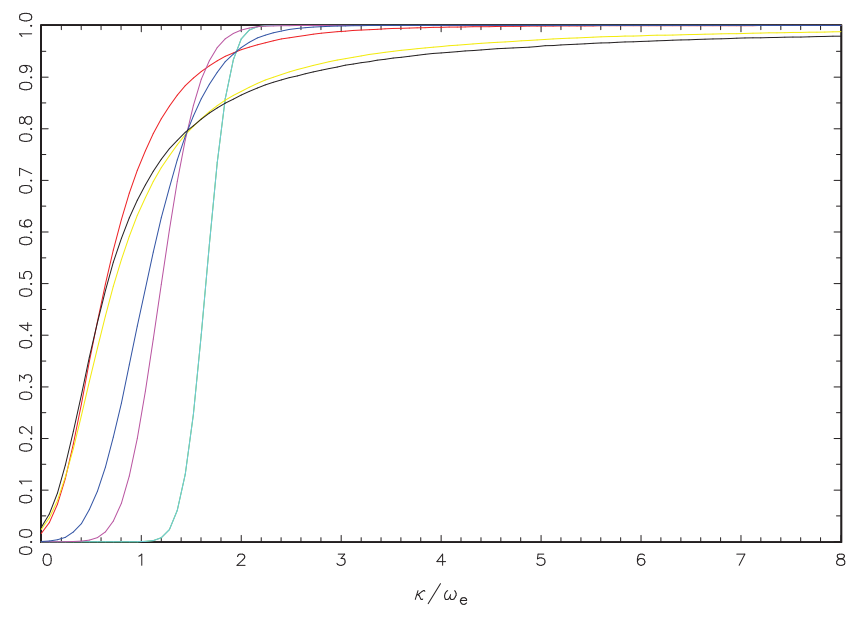

(b) $\bar{\alpha}=15$ $z_{\lambda}, t_{\beta}^{R Q F}:-, z_{\lambda}^{m 2}:-$, Dan- $J_{s}:-$, Dan-J: $\quad, t P S W_{s}^{1}:-, t P S W^{1}:-$

Figure 2. Local Asymptotic Power: I(1) innovations 


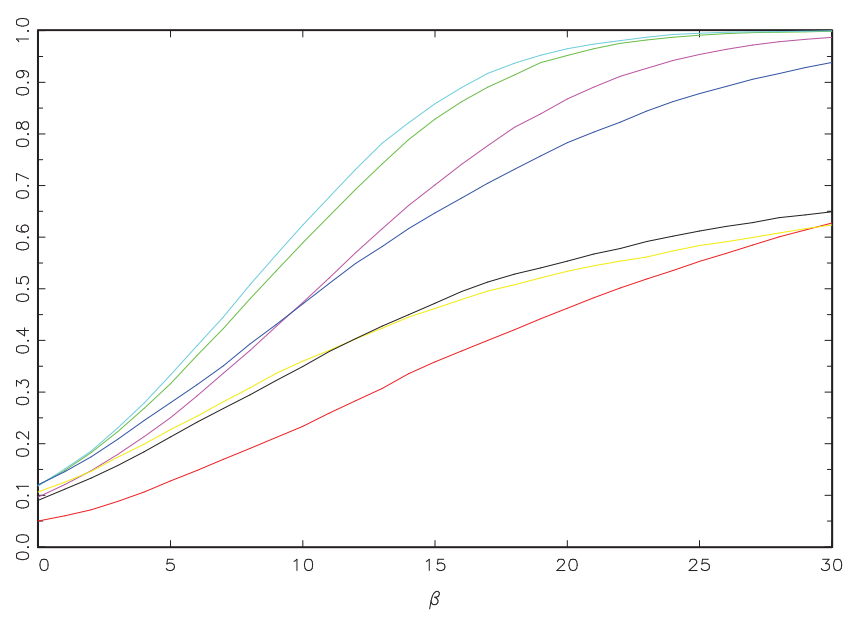

(a) $T=100, \theta=-0.4$

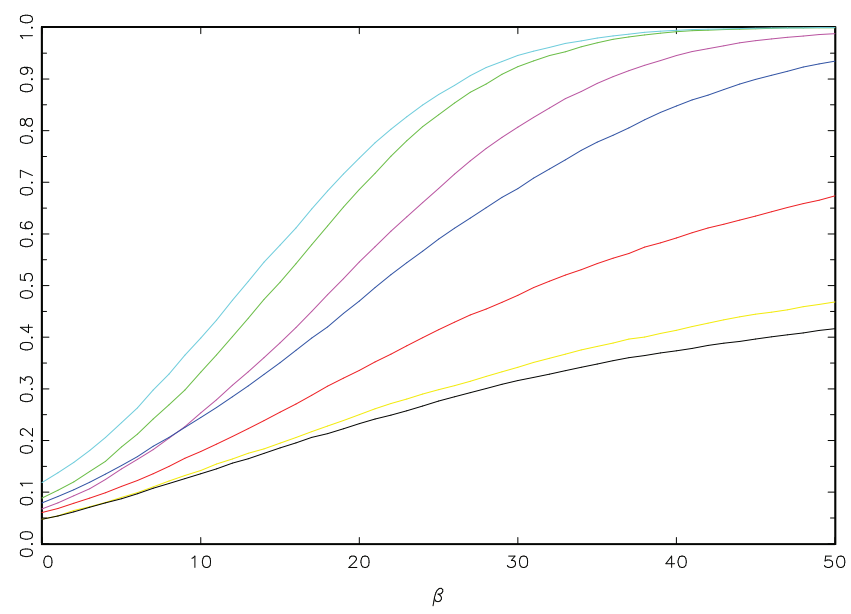

(c) $T=100, \theta=0.0$

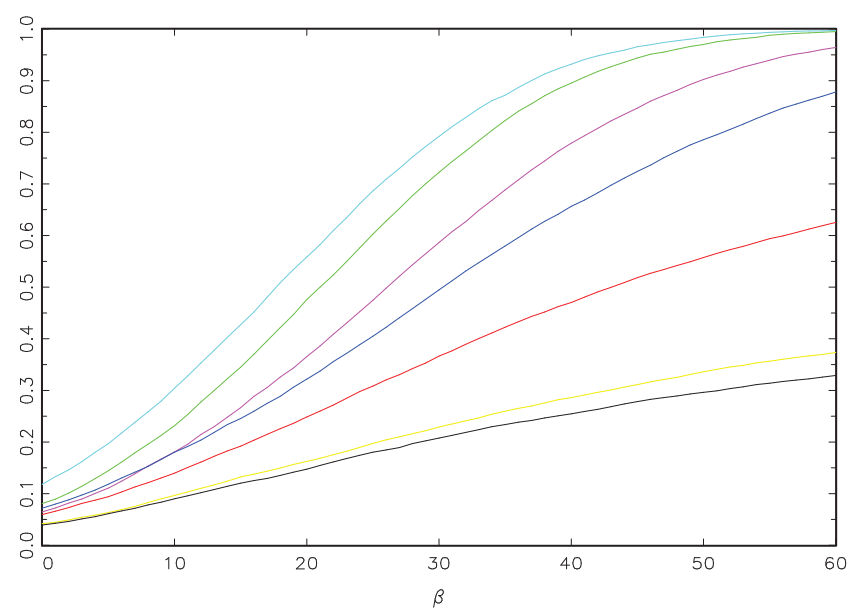

(e) $T=100, \theta=0.4$

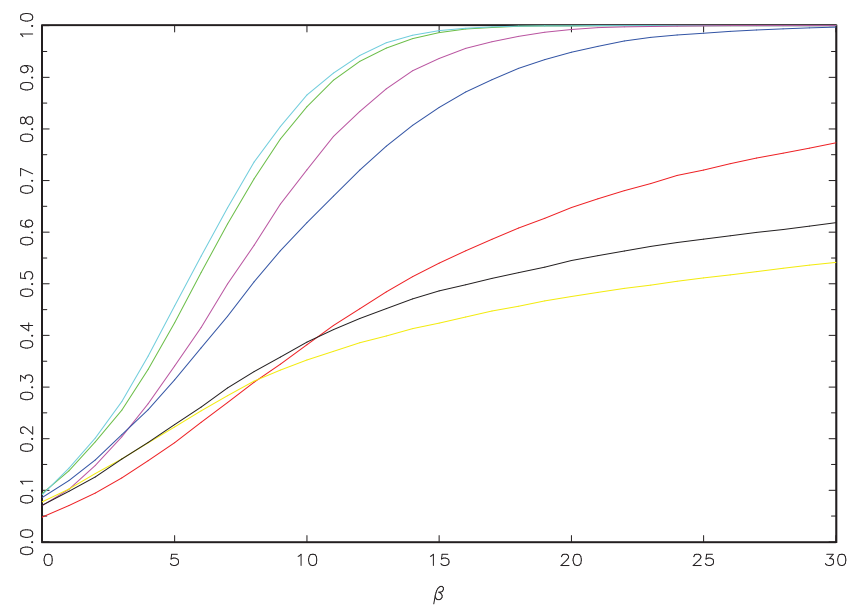

(b) $T=250, \theta=-0.4$

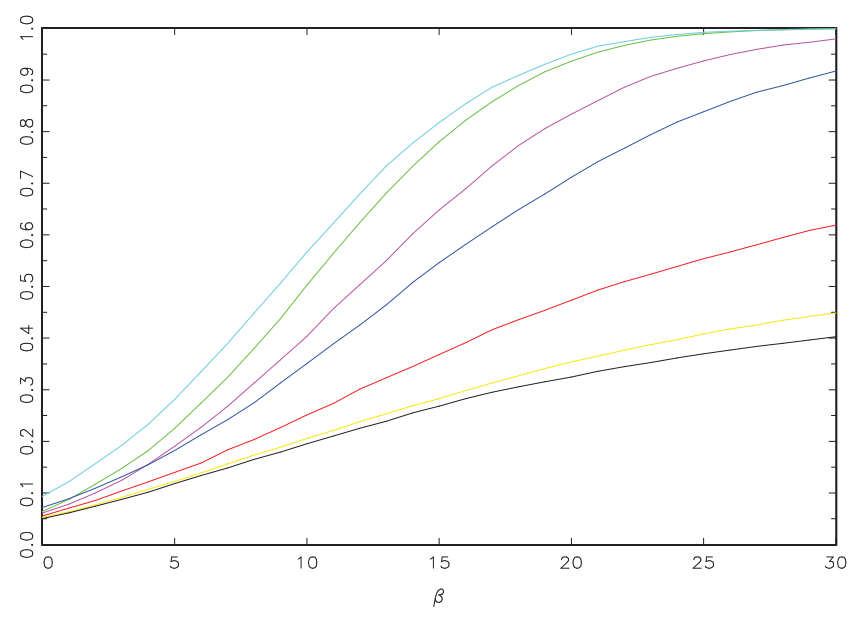

(d) $T=250, \theta=0.0$

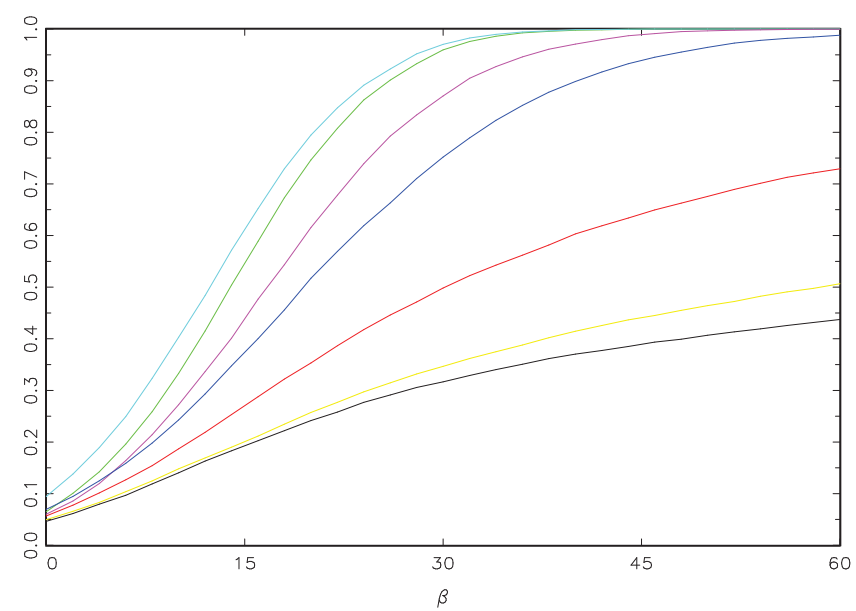

(f) $T=250, \theta=0.4$

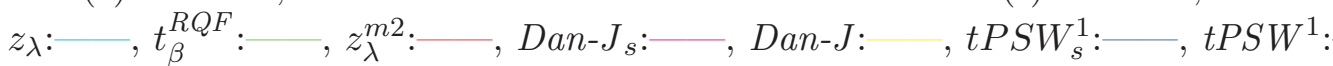

Figure 3. Finite Sample Empirical Power: $\bar{\alpha}=0$ 


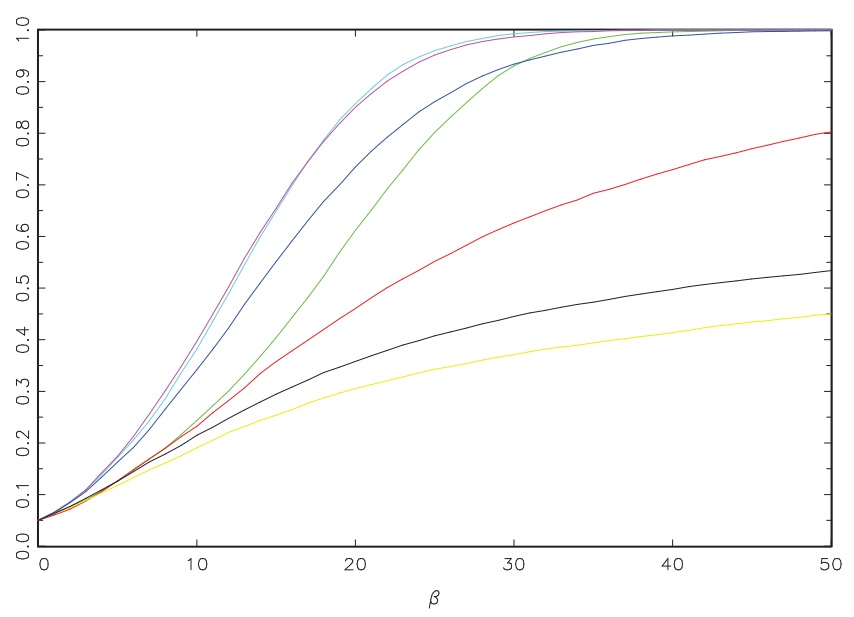

(a) $T=100, \theta=-0.4$

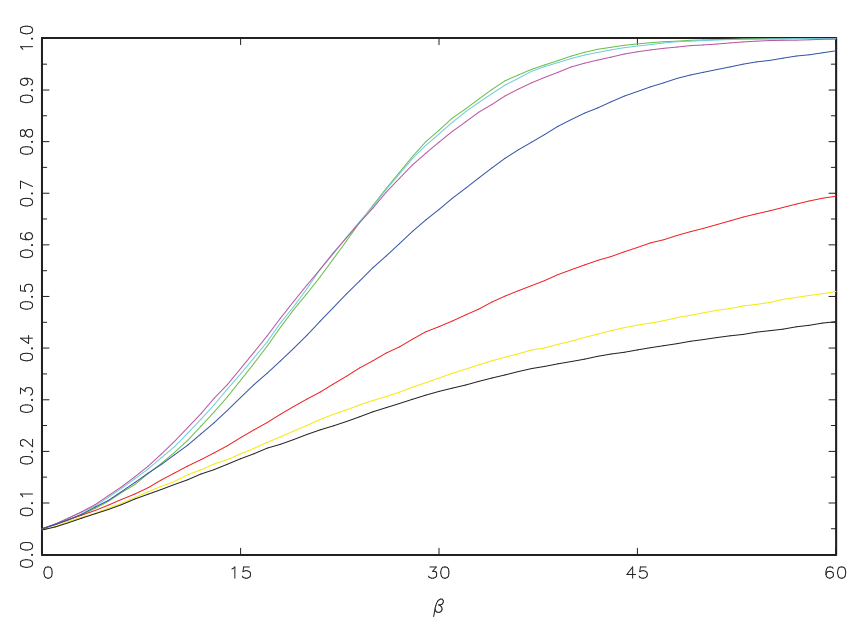

(c) $T=100, \theta=0.0$

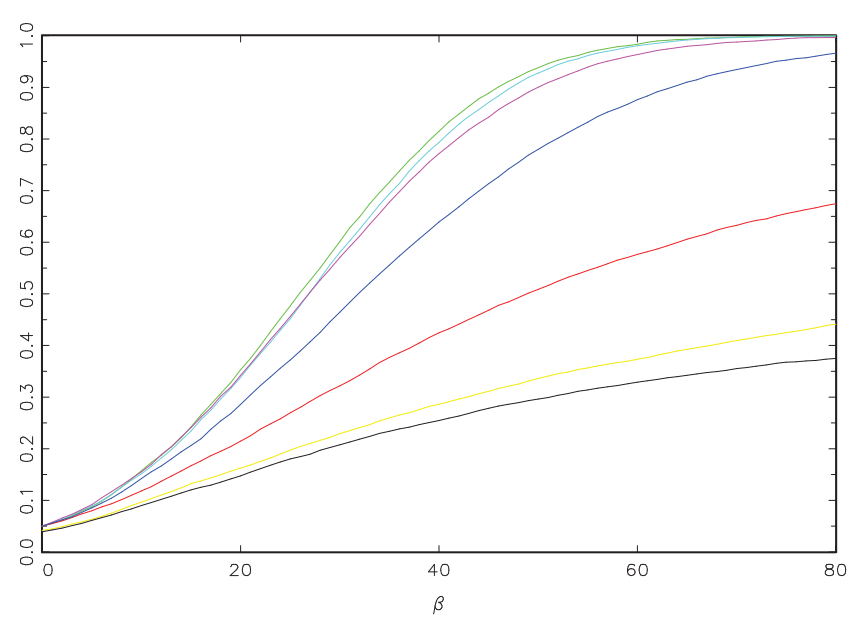

(e) $T=100, \theta=0.4$

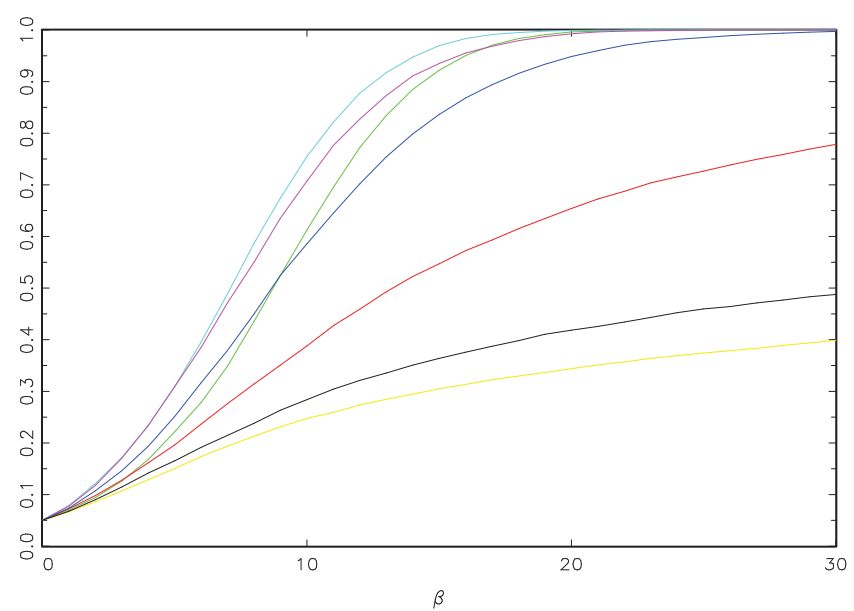

(b) $T=250, \theta=-0.4$

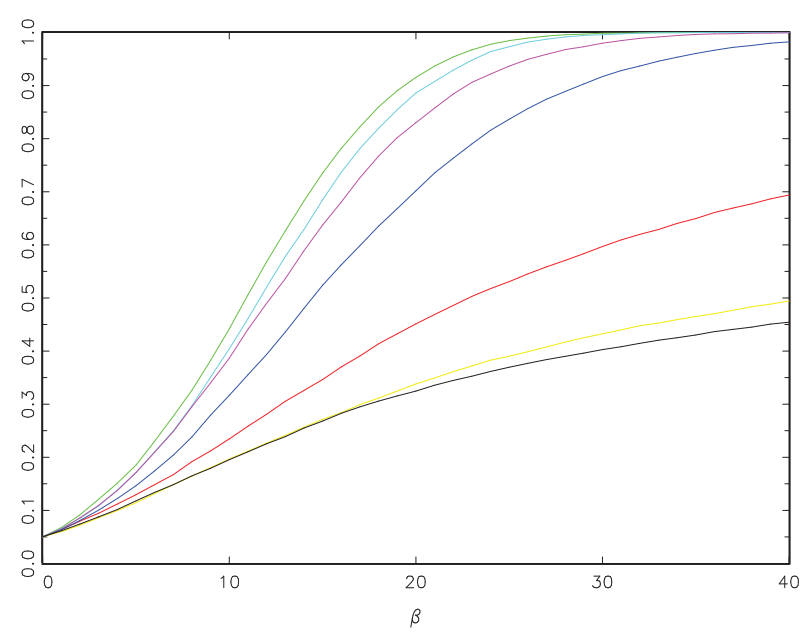

(d) $T=250, \theta=0.0$

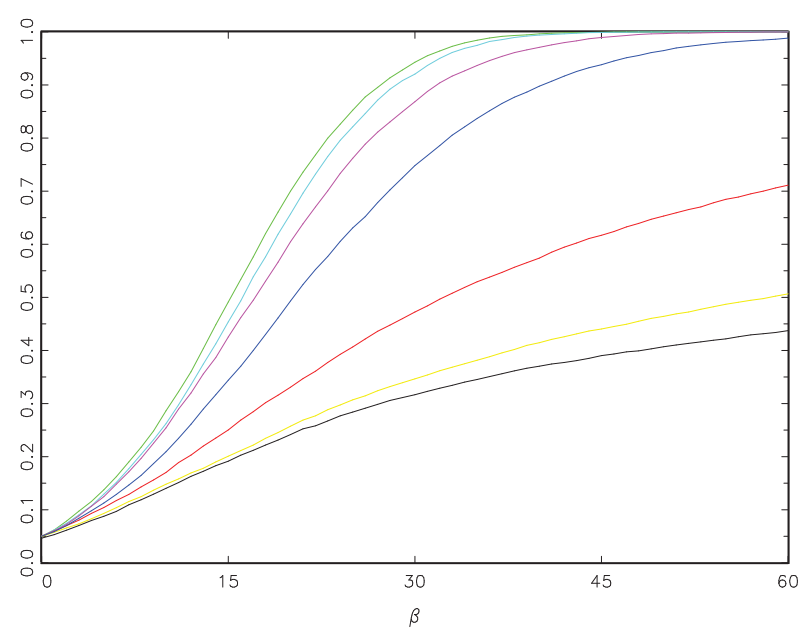

(f) $T=250, \theta=0.4$ $z_{\lambda}:-, t_{\beta}^{R Q F}:-, z_{\lambda}^{m 2}:-, D^{2}-J_{s}:-$, Dan-J: , tPSW $W_{s}^{1}:-, t P S W^{1}:$

Figure 4. Finite Sample Size Adjusted Power: $\bar{\alpha}=0$ 


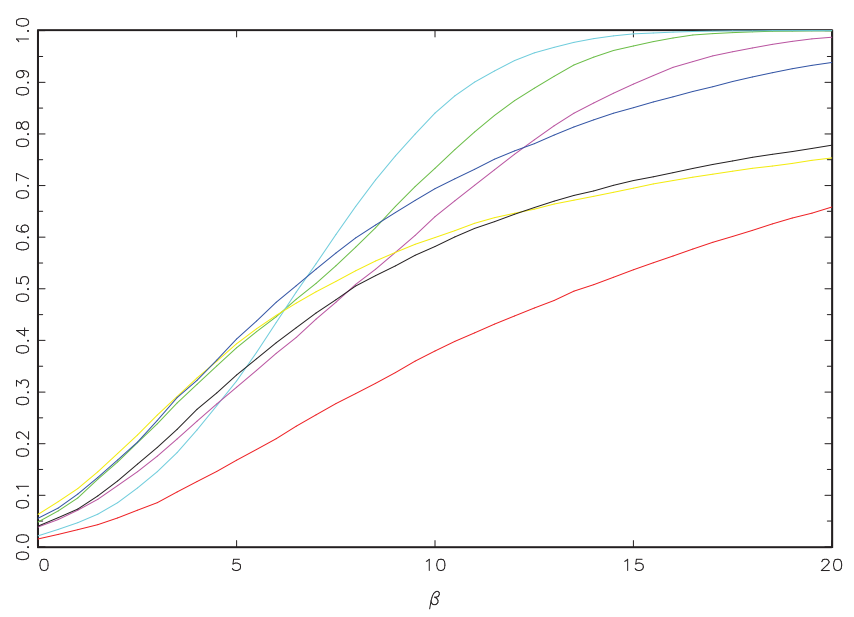

(a) $T=100, \theta=-0.4$

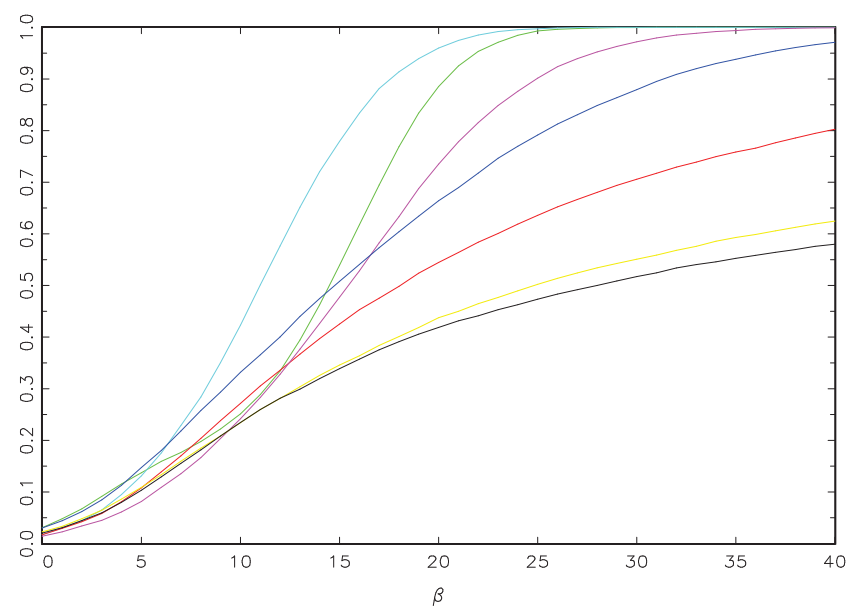

(c) $T=100, \theta=0.0$

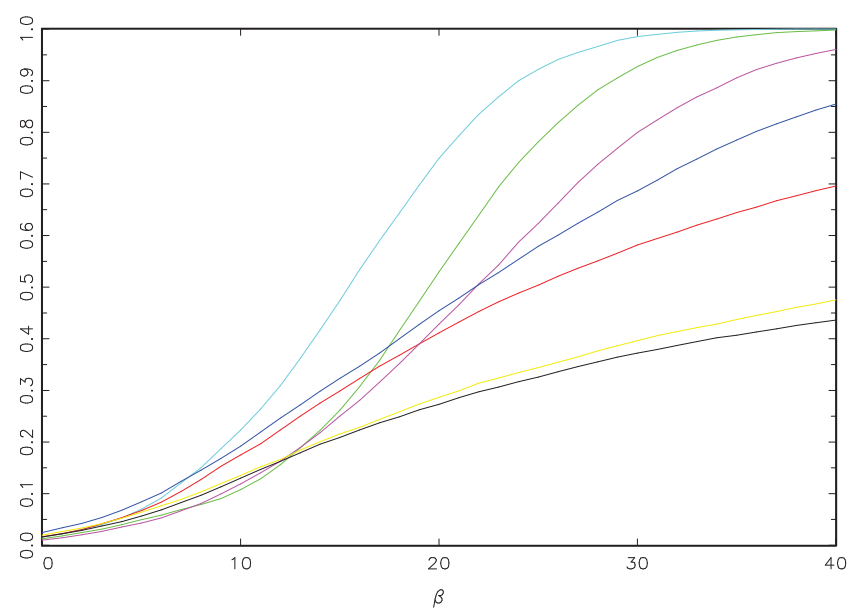

(e) $T=100, \theta=0.4$

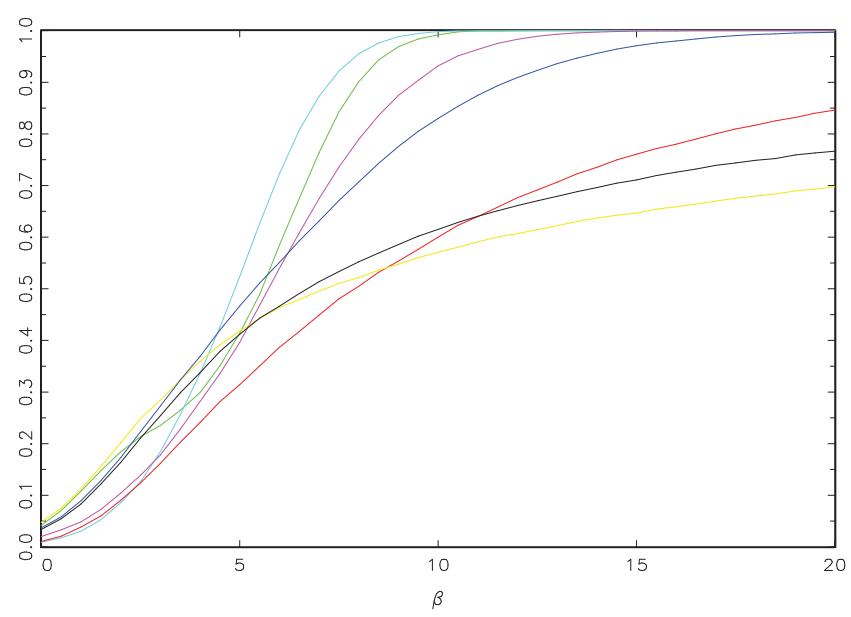

(b) $T=250, \theta=-0.4$

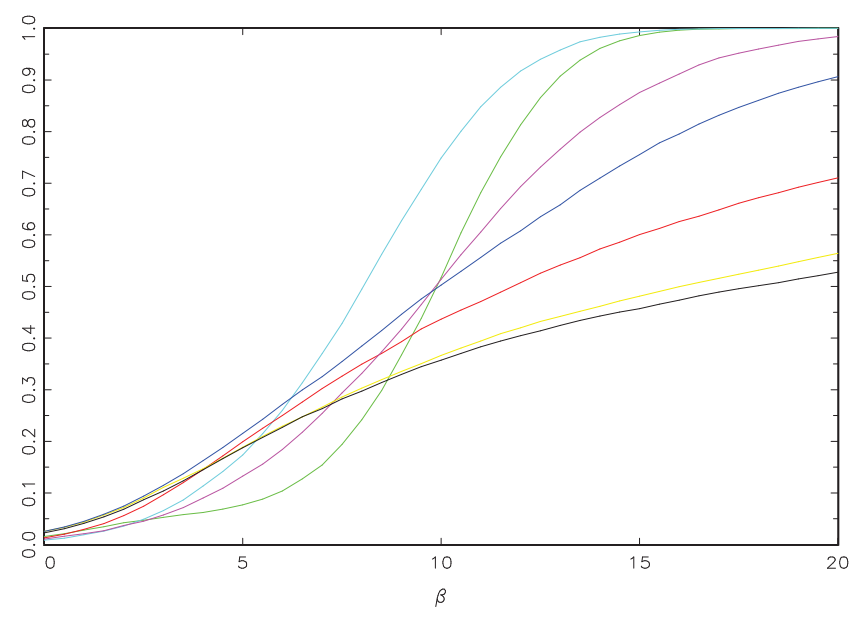

(d) $T=250, \theta=0.0$

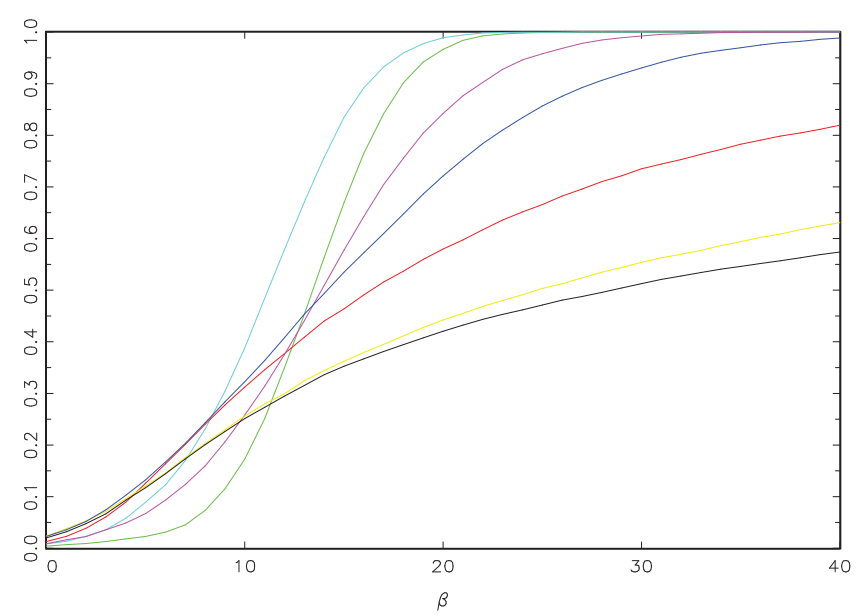

(f) $T=250, \theta=0.4$ $z_{\lambda}:-, t_{\beta}^{R Q F}:-, z_{\lambda}^{m 2}:-, D^{2}-J_{s}:-$, Dan-J: $, t P S W_{s}^{1}:-, t P S W^{1}:$

Figure 5. Finite Sample Empirical Power: $\bar{\alpha}=5$ 


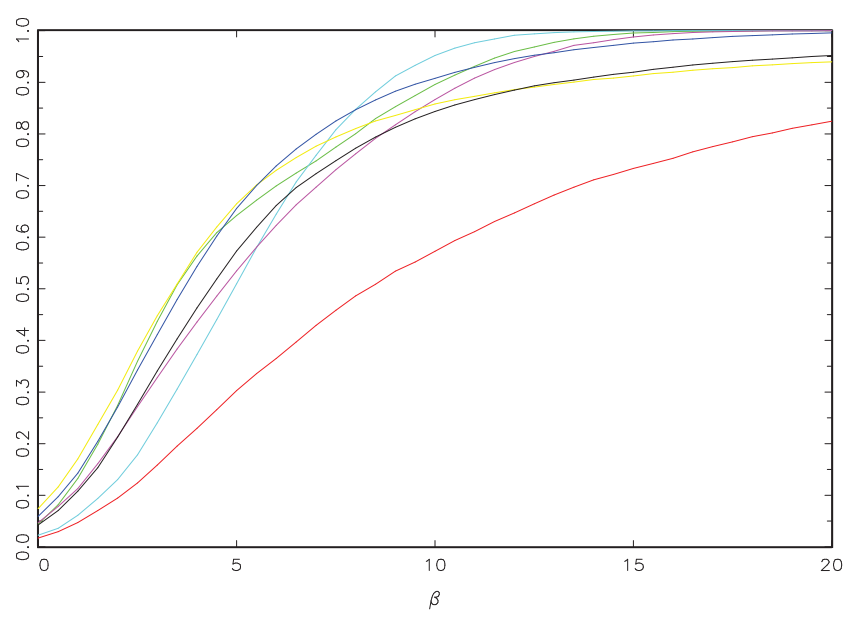

(a) $T=100, \theta=-0.4$

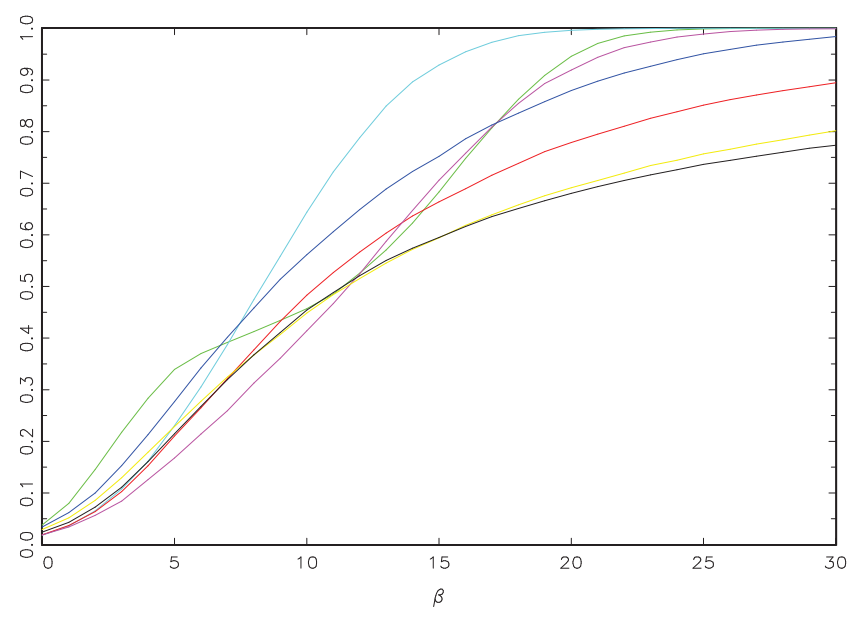

(c) $T=100, \theta=0.0$

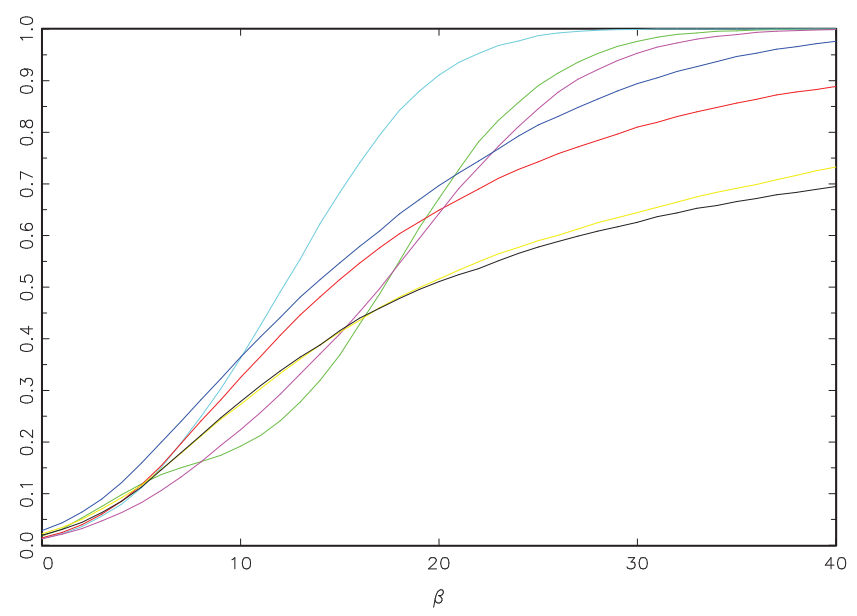

(e) $T=100, \theta=0.4$

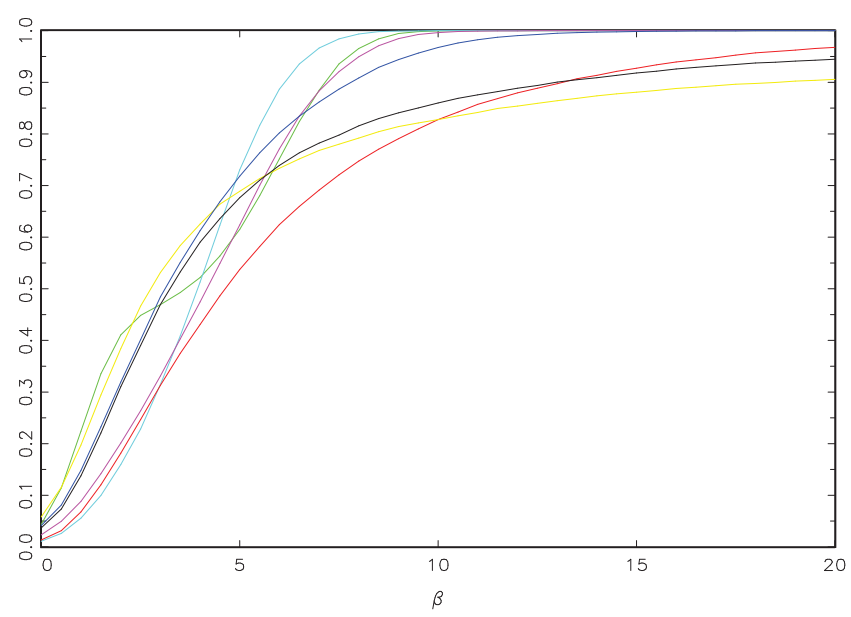

(b) $T=250, \theta=-0.4$

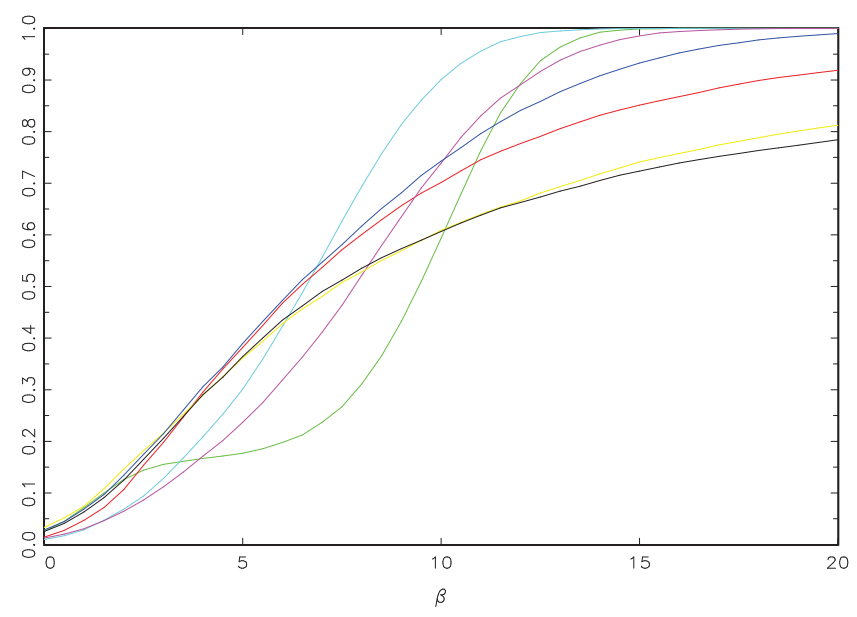

(d) $T=250, \theta=0.0$

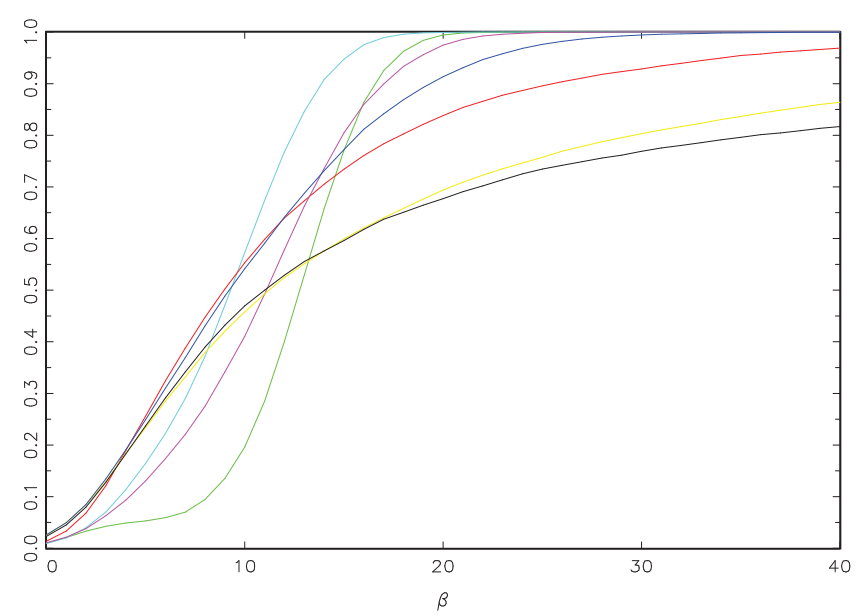

(f) $T=250, \theta=0.4$

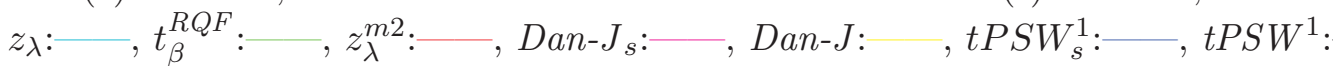

Figure 6. Finite Sample Empirical Power: $\bar{\alpha}=10$ 


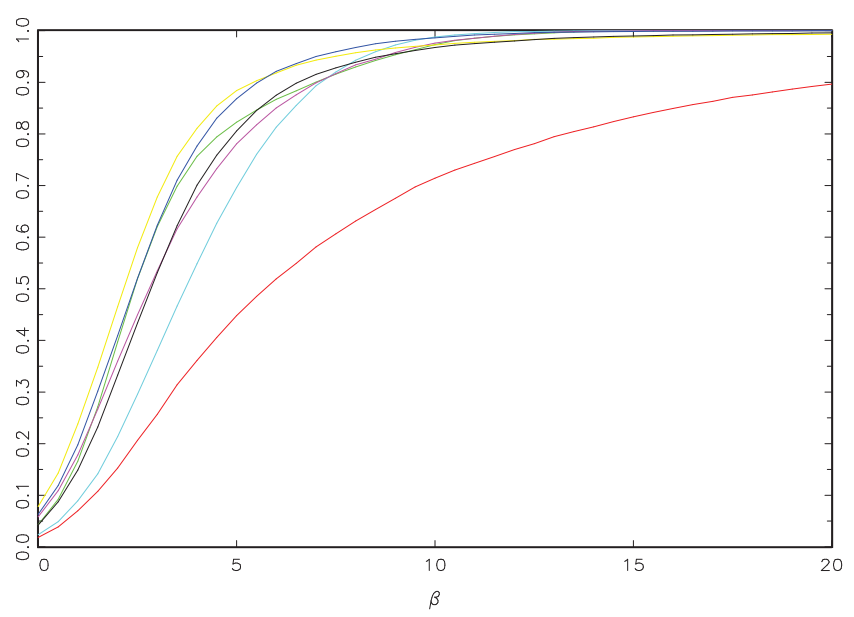

(a) $T=100, \theta=-0.4$

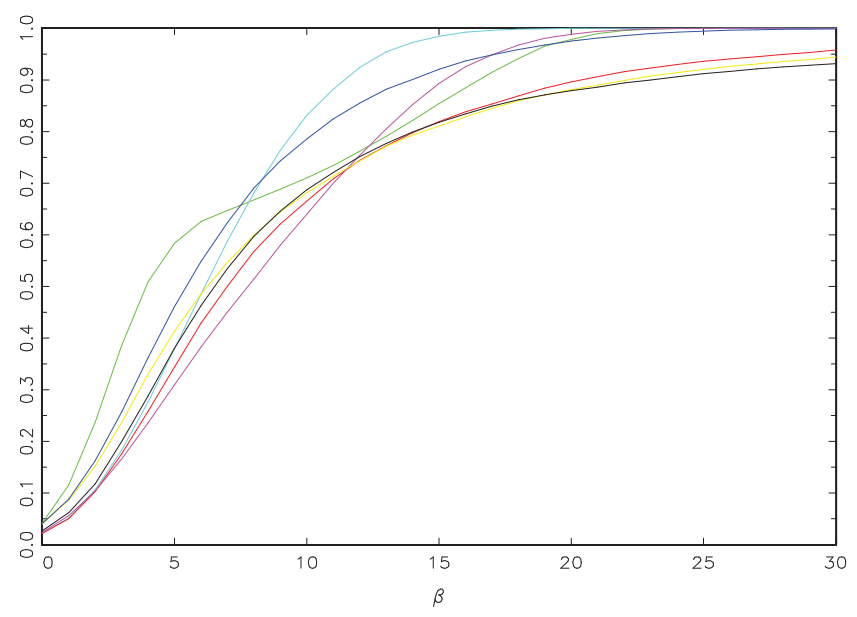

(c) $T=100, \theta=0.0$

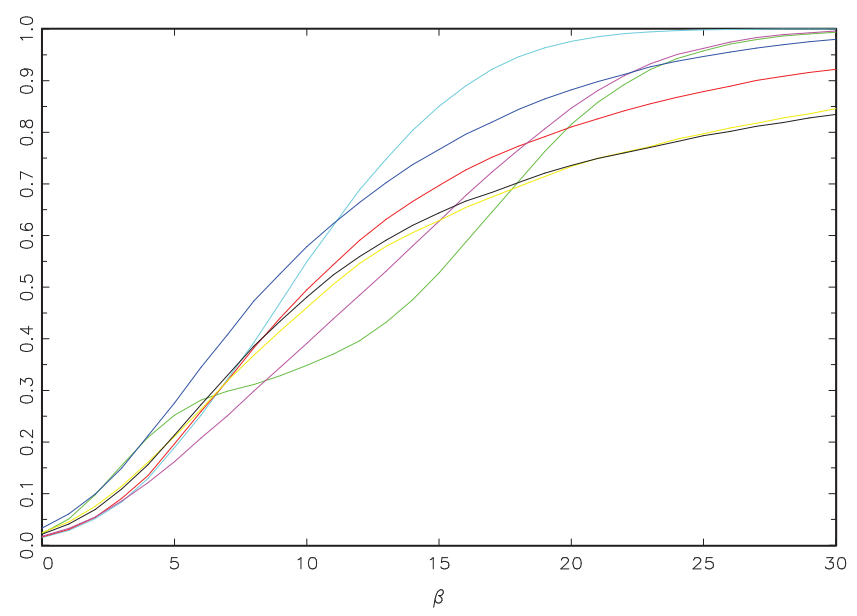

(e) $T=100, \theta=0.4$

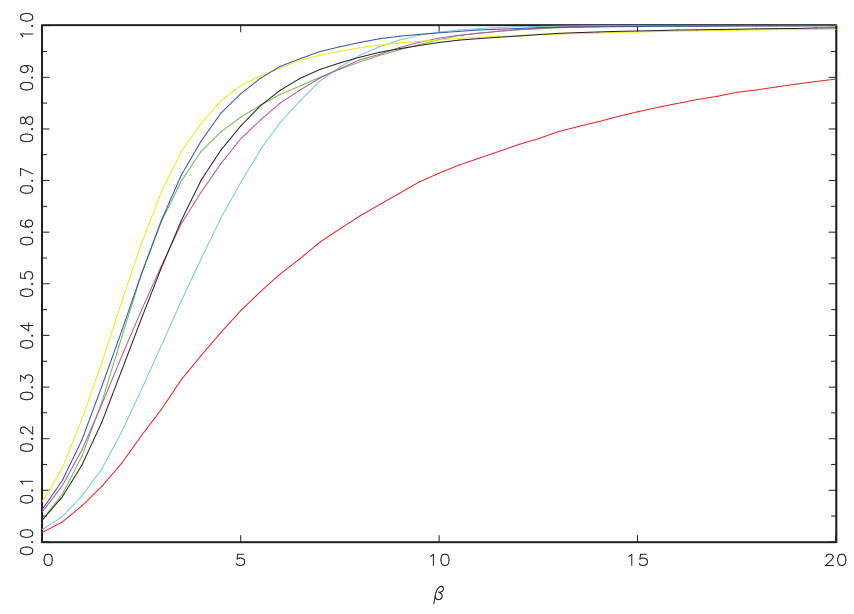

(b) $T=250, \theta=-0.4$

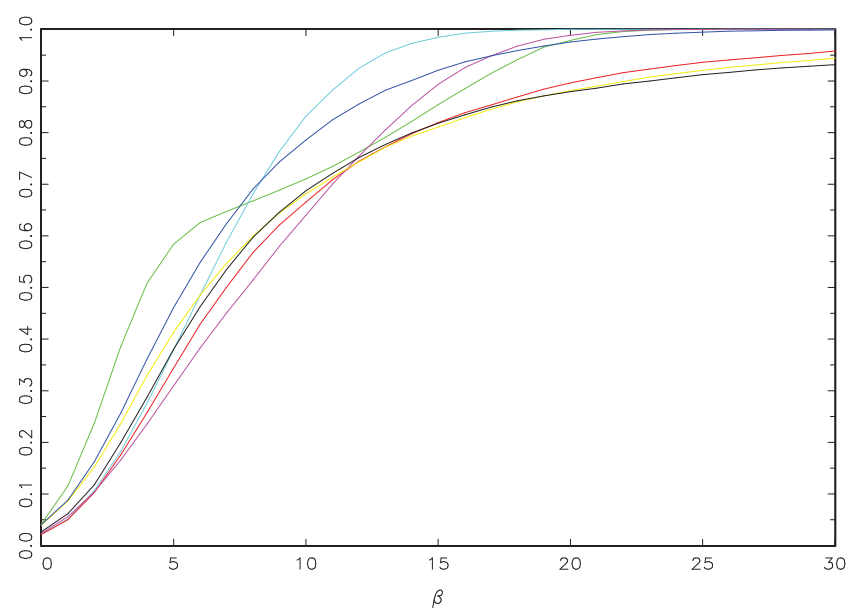

(d) $T=250, \theta=0.0$

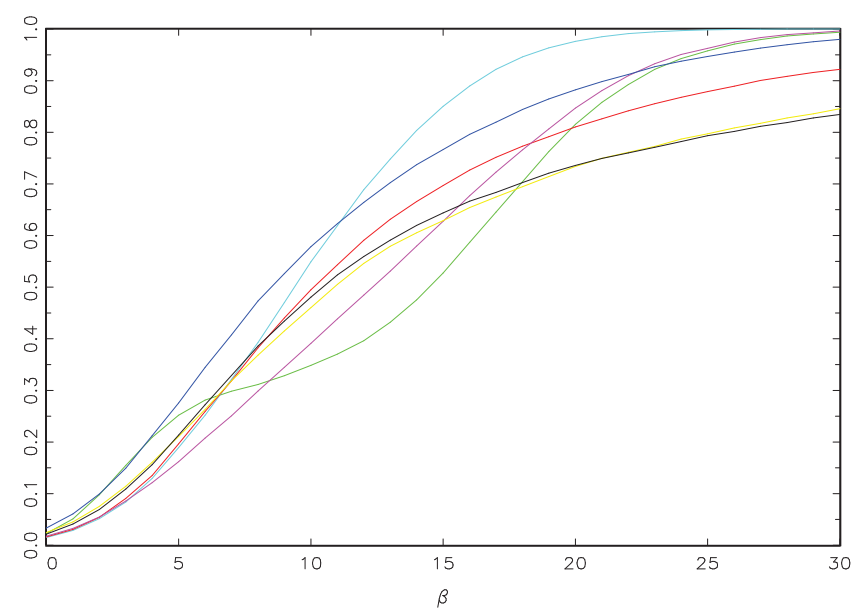

(f) $T=250, \theta=0.4$ $z_{\lambda}:-\quad, t_{\beta}^{R Q F}:-, z_{\lambda}^{m 2}:-, D^{2}-J_{s}:-$, Dan-J: , $t P S W_{s}^{1}:-, t P S W^{1}:$

Figure 7. Finite Sample Empirical Power: $\bar{\alpha}=15$ 

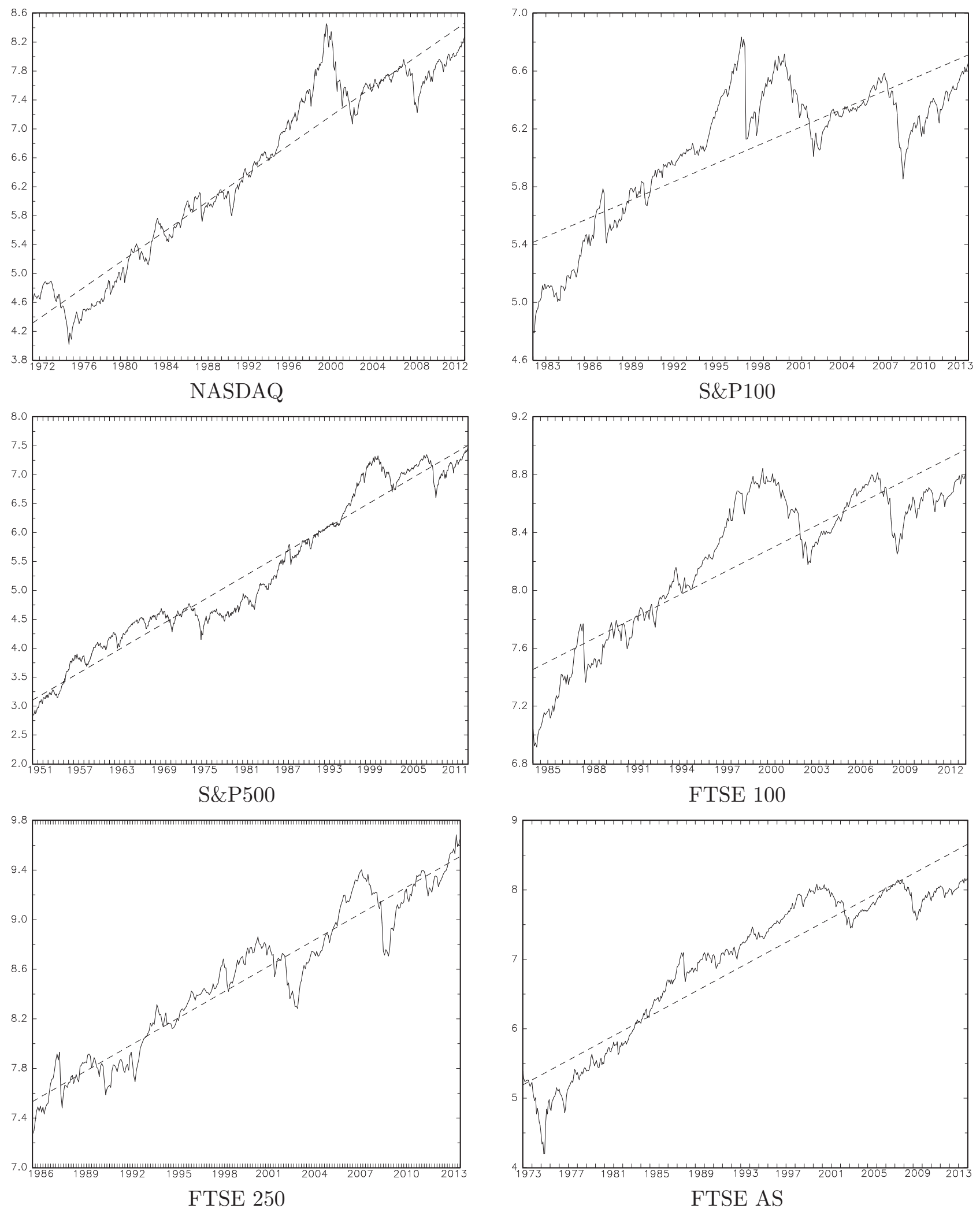

Index: — , Fitted Series: - - -

Figure 8. Trends Fitted to Equity Indices 

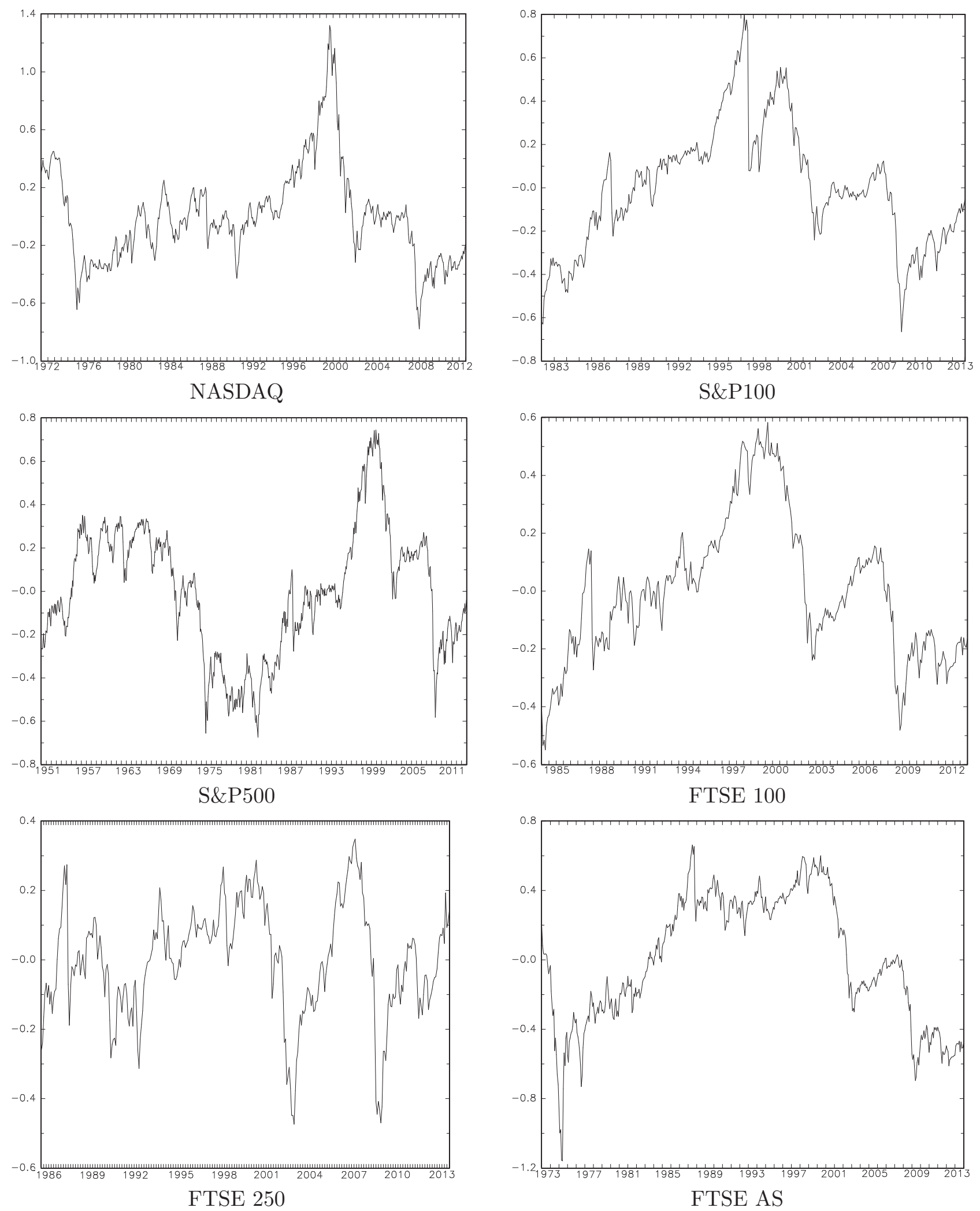

Figure 9. Detrended Equity Indices 